\title{
Glycine transporter-1 antagonist provides 1 neuroprotection in vivo
}

\section{Richard Bergeron ( $\nabla$ rbergeron@ohri.ca )}

University of Ottawa

Julia Cappelli

University of Ottawa

\section{Pamela Khacho}

University of Ottawa

\section{Boyang Wang}

University of Ottawa

\section{Alexandra Sokolovski \\ University of Ottawa \\ Wafae Bakkar \\ Ottawa Hospital Research Institute}

\section{Sophie Raymond}

University of Ottawa

\section{Nina Ahlskog}

University of Ottawa

\section{Julian Pitney}

University of Ottawa

Junzheng Wu

University of Ottawa

Prakash Chudalayandi

University of Ottawa

\section{Adrian Wong}

University of Ottawa

\section{Article}

Keywords: Glutamate, D-serine, serine racemase (SR), glycine transporter type 1 (GlyT1), glycine transporter type 1 antagonist (GlyT1-A), NFPS, glycine oxidase (GO), oxygen-glucose deprivation (OGD), dynamin, dynasore, immunohistochemistry, sniffer-patch technique, light sheet fluorescence microscopy (LSFM), tissue clearing, Laser Doppler flowmetry, adhesive removal test, whole-cell patch-clamp 
technique, ischemia, stroke, photothrombosis, endothelin-1, GlyT1+/- mouse, SR-/- mouse, GluN2A-/mouse, hippocampus, Al/AIVIA 9

Posted Date: May 11th, 2021

DOI: https://doi.org/10.21203/rs.3.rs-460311/v1

License: (c) (1) This work is licensed under a Creative Commons Attribution 4.0 International License. Read Full License 


\section{Authors:}

Julia Cappelli (jcapp082@uottawa.ca) ${ }^{1 *}$, Pamela Khacho (pkhac024@uottawa.ca) ${ }^{1 *}$, Boyang Wang (jwang149@uottawa.ca)1, Alexandra Sokolovski (asoko047@uottawa.ca)1, Wafae Bakkar (wafae.bakkar@hc-sc.gc.ca) ${ }^{2}$, Sophie Raymond (sraym054@uottawa.ca)1, Nina Ahlskog (nahlskog@gmail.com), Julian Pitney (julianpitney@gmail.com)1, Junzheng Wu (Junzheng@gmail.com) ${ }^{1}$, Prakash Chudalayand (pchudala@uottawa.ca)1, Adrian Y.C. Wong (awon2@uottawa.ca) ${ }^{1}$, Richard Bergeron (rbergeron@ohri.ca) ${ }^{1}$

$10 *$ these authors contributed equally to this work

\section{Affiliations:}

${ }^{1}$ Cellular and Molecular Medicine Department, University of Ottawa, 451 Smyth Road, Roger Guindon Building, Room 3501N, Ottawa, Ontario, Canada, K1H 8M5. Tel: (613) 562-5800 Extension 3296, Fax: (613) 562-5278; ${ }^{2}$ Ottawa Hospital Research Institute, 451 Smyth Road, Roger Guindon Building, Room 3501N, Ottawa, Ontario, Canada, K1H 8M5. Tel: (613) 562-5800 ext. 3296

\section{Running title:}

20 Glycine-induced NMDAR internalization.

\section{Keywords:}

Glutamate, D-serine, serine racemase (SR), glycine transporter type 1 (GlyT1), glycine transporter type 1 antagonist (GlyT1-A), NFPS, glycine oxidase (GO), oxygen-glucose deprivation (OGD), dynamin, dynasore, immunohistochemistry, sniffer-patch technique, light sheet fluorescence microscopy (LSFM), tissue clearing, Laser Doppler flowmetry, adhesive removal test, whole-cell patch-clamp technique, ischemia, stroke, photothrombosis, endothelin-1, GlyT1 ${ }^{+/-}$mouse; $\mathrm{SR}^{-/-}$ mouse, GluN2 $\mathrm{A}^{-/}$mouse, hippocampus, AI/AIVIA 9

\section{1}

Figures and Extended Data

Number of Figures: 4

Number of Extended Data Figures/Tables: 10 


\begin{tabular}{|c|c|c|}
\hline 33 & \multicolumn{2}{|c|}{ Abbreviations: } \\
\hline 35 & AAV: & Adeno associated virus; \\
\hline 36 & ACSF: & Artificial cerebrospinal fluid; \\
\hline 37 & CHO: & Chinese Hamster Ovary; \\
\hline 38 & CPA: & Cyclopiazonic acid; \\
\hline 39 & CV: & Cresyl violet; \\
\hline 40 & DBP: & Dynamin blocking peptide; \\
\hline 41 & $\mathrm{ddH}_{2} \mathrm{O}$ : & Double distilled water; \\
\hline 42 & EPSCs: & Excitatory postsynaptic currents; \\
\hline 43 & ET-1: & Endothelin-1; \\
\hline 44 & FJC: & FluoroJade C; \\
\hline 45 & GBS: & Glycine binding site; \\
\hline 46 & GINI: & Glycine-induced NMDAR internalization; \\
\hline 47 & GluN2A $A^{-/-}$: & NMDAR GluN2A subunit knockout; \\
\hline 48 & GlyR: & Glycine receptor; \\
\hline 49 & GlyT1: & Glycine transporter type 1; \\
\hline 50 & GlyT1 $^{+/-}:$ & Heterozygous glycine transporter type 1 ; \\
\hline 51 & GlyT1-A: & Glycine transporter type 1 antagonist; \\
\hline 52 & GO: & Glycine oxidase; \\
\hline 53 & i.p.: & Intraperitoneal; \\
\hline 54 & LDF: & Laser Doppler flowmetry; \\
\hline 55 & LSFM: & Light sheet fluorescence microscopy; \\
\hline 56 & TMCAO & Transient middle cerebral artery occlusion; \\
\hline 57 & MRI: & Magnetic resonance imaging; \\
\hline 58 & NFPS: & $N$-[3-(4'-fluorophenyl)-3-(4'-phenylphenoxy)propyl]sarcosine; \\
\hline 59 & NMDAR: & $N$-methyl-D-aspartate receptor; \\
\hline 60 & NMDG: & $\mathrm{N}$-methyl-D-glucamine; \\
\hline 61 & OGD: & Oxygen-glucose deprivation paradigm; \\
\hline 62 & PBSG: & $0.25 \%(\mathrm{w} / \mathrm{v})$ gelatin in PBS; \\
\hline 63 & PBSGT: & $0.25 \%(\mathrm{w} / \mathrm{v})$ gelatin and $0.2 \%$ Triton $\mathrm{X}-100(\mathrm{v} / \mathrm{v})$ in PBS; \\
\hline 64 & PFA: & Paraformaldehyde; \\
\hline 65 & Popen: & Open probability; \\
\hline 66 & PT: & Photothrombosis; \\
\hline 67 & RT: & Room temperature; \\
\hline 68 & $\mathbf{S R}^{-/}:$ & Serine racemase knockout; \\
\hline 69 & TTC: & 2,3,5-triphenyltetrazolium chloride; \\
\hline 70 & VGAT: & Vesicular GABA transporter; \\
\hline 71 & VGLUT: & Vesicular glutamate transporter; \\
\hline 72 & WT: & Wild type. \\
\hline
\end{tabular}


73 Glycine fulfills several roles in biology including protein synthesis, inhibitory transmission

74 via glycine receptor activation and excitatory transmission through glutamate-sensitive $\mathrm{N}$ 75 methyl-D-aspartate receptors (NMDARs). Low glycine doses enhance NMDAR function 76 while high doses trigger glycine-induced NMDAR internalization (GINI) in vitro. The 77 physiological relevance of GINI has been questioned given that the high-affinity glycine transporter type 1 (GlyT1), located on astrocytes and neurons, maintains synaptic glycine 79 concentrations far below the level that would saturate the glycine binding site (GBS) on 80 NMDARs. Here, we report evidence that GINI occurs also in vivo and is neuroprotective 81 following ischemic insult. Mice pre-treated with a GlyT1 antagonist (GlyT1-A), which 82 increased glycine levels, exhibited smaller stroke volume, reduced cell death, and minimized 83 behavioural deficits following stroke induction by either photothrombosis or endothelin-1. 84 We demonstrate that in a modified in vitro ischemic paradigm, glycine is released at levels 85 surpassing what occurs during ischemia alone. Therefore, glycine accumulates in the 86 synaptic cleft, enhances occupancy of GBS and reaches the set point to trigger GINI. We 87 report that GINI is observed during stroke, in vivo, only in the presence of a GlyT1-A. 88 Moreover, we show evidence of a protective effect on the vasculature in the peri-infarct area. 89 Therefore, these data strongly suggest that GlyT1 is a therapeutic target to prevent cell death 90 following an ischemic event. 
Introduction

92 Neuronal death following ischemic stroke is triggered by uncontrolled glutamate release leading

93 to the overactivation of $N$-methyl-D-aspartate receptors (NMDARs) on surrounding neurons, and

94 in turn induces excessive $\mathrm{Ca}^{2+}$-influx primarily through NMDARs ${ }^{1}$. In physiological conditions

95 NMDARs require glutamate binding on the GluN2 subunit and glycine binding on the glycine

96 binding site (GBS) on the GluN1 subunit ${ }^{2}$. Ascher's group showed that glycine ${ }^{3}$, or a glycine-like

97 substance ${ }^{4}$, is a required co-agonist for NMDAR activation. Moreover, Salter and co-workers

98 reported that high doses of glycine trigger GINI, in vitro, by promoting endocytosis of NMDAR

99 through clathrin/dynamin-dependent machinery ${ }^{5}$. Unlike constitutive internalization, which

100 requires no channel activation ${ }^{5,6}$, NMDAR internalization following glycine "priming" requires

101 both glutamate and glycine present in the synaptic $\mathrm{cleft}^{5}$. We found that during an oxygen/glucose

102 deprivation paradigm, in vitro, not only glutamate but also an excess of glycine is released in the

103 extracellular space. However, this is not sufficient to trigger GINI since the level of extracellular

104 glycine is buffered by the glycine transporter $1(\mathrm{GlyT} 1)^{7-10}$. We report that GlyT1 antagonist

105 (GlyT1-A) administration before an ischemic event, in vivo, triggers GINI and provides

106 neuroprotection.

107

108 Results

109 High concentrations of glycine induce NMDAR internalization

110 We first determined the effects of bath application of increasing glycine concentrations on 111 stimulation-evoked NMDAR excitatory postsynaptic currents (NMDAR-EPSCs) recorded from

112 CA1 pyramidal neurons from acute hippocampal brain slices (Fig. 1a). At glycine concentrations 113 below $250 \mu \mathrm{M}$, NMDAR-ESPC amplitudes were potentiated in a dose-dependent fashion ${ }^{3,10-12}$.

114 However, increasing the glycine concentration to $1 \mathrm{mM}$ resulted in a significant decrease in 115 NMDAR-EPSC amplitude ${ }^{5,13}$ and this effect was reversible (Fig. 1b). To verify that this decrease 116 in amplitude was due to GINI, we applied $1 \mathrm{mM}$ glycine in the presence of $100 \mu \mathrm{M}$ dynasore, a 117 cell-permeable inhibitor of both dynamin-1 and dynamin-2, which blocks internalization ${ }^{6,14}$. We 118 found that the decrease in NMDAR-EPSC amplitudes by $1 \mathrm{mM}$ glycine was abolished in the 119 presence of dynasore (Fig. 1c).

120

121 Calcium influx is required for GINI to occur 
122 Previous studies have reported that the activity of dynamin is regulated by the $\mathrm{Ca}^{2+}$-sensitive

123 phosphatase calcineurin ${ }^{15,16}$. Therefore, we explored the role of extracellular and intracellular $\mathrm{Ca}^{2+}$ 124 on NMDAR-EPSC amplitudes in the presence of $1 \mathrm{mM}$ glycine. We examined the effects of 125 various external $\mathrm{Ca}^{2+}$ concentrations on the NMDAR response to $1 \mathrm{mM}$ glycine application. When $1261 \mathrm{mM}$ glycine was applied with low $\mathrm{Ca}^{2+}(1 \mathrm{mM})$, an increase in NMDAR-EPSC amplitude was 127 observed, in contrast to what occurred with normal $\mathrm{Ca}^{2+}$ concentrations $(3.5 \mathrm{mM})$. To further 128 confirm the role of $\mathrm{Ca}^{2+}$ in GINI, we included a $\mathrm{Ca}^{2+}$ chelator BAPTA $(10 \mathrm{mM})$, in the recording 129 electrode. Here, we observed a significant attenuation in the decrease in NMDAR-EPSC amplitude 130 induced by $1 \mathrm{mM}$ exogenous glycine. Moreover, extracellular application of $20 \mu \mathrm{M}$ nimodipine, an 131 L-type $\mathrm{Ca}^{2+}$ channel blocker, also attenuated GINI compared to control, further corroborating the 132 data acquired with BAPTA. In contrast, depleting intracellular $\mathrm{Ca}^{2+}$ stores by incubating 133 hippocampal slices for $1 \mathrm{hr}$ in $30 \mu \mathrm{M}$ cyclopiazonic acid (CPA), an inhibitor of intracellular $\mathrm{Ca}^{2+}$ 134 pumps, had no effect on the glycine-induced decrease in NMDAR-EPSC amplitude (Fig. 1d). 135 Together these data suggest that external $\mathrm{Ca}^{2+}$ influx across the plasma membrane is required for 136 GINI to occur.

\section{Genetic elevation of extracellular glycine facilitates GINI}

139 Heterozygous glycine transporter type $1\left(\mathrm{GlyT}^{+/}\right)$mice exhibit a higher level of endogenous 140 extracellular glycine ${ }^{17,18}$. Therefore, we hypothesized that in these mice GINI could be triggered 141 by lower doses of glycine. As illustrated in figure 1e, while there was no significant effect in the 142 NMDAR-EPSC amplitude following bath application of $10 \mu \mathrm{M}$ or $1 \mathrm{mM}$ glycine between wild type 143 (WT) and $\mathrm{GlyT}^{+/}$mice, bath application of $250 \mu \mathrm{M}$ glycine, which potentiated the NMDAR-EPSC 144 amplitude in WT mice, significantly inhibited the NMDAR-EPSC amplitude in $\mathrm{GlyT}^{+/-}$mice 145 (Fig. 1f). The decrease of the NMDAR-EPSC amplitude induced by $250 \mu \mathrm{M}$ glycine in $\mathrm{GlyT}^{+/-}$ 146 mice was abolished in the presence of dynasore (Fig. 1g). Therefore, the high levels of endogenous 147 glycine in the $\mathrm{GlyT}^{+/ /}$mice trigger GINI at lower exogenous glycine concentrations.

\section{Role of glycine binding site occupancy}

150 In addition to glycine, D-serine, also activates the GBS ${ }^{4,19}$. As illustrated in figure $1 \mathrm{~h}$, the effects 151 of increasing D-serine concentrations on NMDAR-EPSC amplitudes in acute slices from WT mice 152 was also dose-dependent. Moreover, the dose-response curve of NMDAR-EPSC amplitudes to D- 
153 serine was left-shifted relative to that of glycine due to its higher affinity to the GBS ${ }^{20}$ (Fig. 1i).

154 The decrease in NMDAR-EPSC amplitude evoked following bath application of $1 \mathrm{mM}$ D-serine 155 was also abolished in the presence of dynasore (Fig. 1j).

157 We next investigated whether GINI could be modulated by increasing levels of either glycine or 158 D-serine in WT mice. Glycine levels were increased via bath application of the selective GlyT1159 A, $\quad N$-[3-(4'-fluorophenyl)-3-(4'-phenylphenoxy)propyl]sarcosine (NFPS; 300nM) ${ }^{21-25}$. As 160 expected, there was a significant increase in evoked NMDAR-EPSC amplitude in the presence of 161 NFPS alone ${ }^{10}$. However, when NFPS was applied together with a potentiating concentration of D162 serine $(10 \mu \mathrm{M})$, a significant decrease in NMDAR-EPSC amplitude was observed (Fig. 1k). 163 Interestingly, when NMDARs were first primed with high doses of glycine or D-serine, a 164 subsequent application of a low dose of glycine or D-serine, also induced GINI (Extended Data 165 Fig. 1).

167 Next, we used a transgenic mouse model in which the serine racemase gene was knocked out (SR ${ }^{-}$ $168{ }^{\prime-}$ mice $)^{26-28}$, as these mice exhibit low levels of D-serine. In both WT and $\mathrm{SR}^{-/}$mice, a low dose of 169 D-serine $(10 \mu \mathrm{M})$ potentiated the evoked NMDAR-EPSC amplitude. However, SR ${ }^{-/-}$mice required 170 a higher dose of D-serine $(2 \mathrm{mM})$ than WT mice $(1 \mathrm{mM})$ to induce a decrease in NMDAR-EPSC 171 amplitude (Fig. 11-m). These findings suggest that there is a common mechanism of action for 172 glycine or D-serine to trigger GINI. Additionally, we found that GINI was not subunit-specific 173 (Extended Data Fig. 2a-e), nor attributed to AMPA receptor activity (Extended Data Fig. 2f), and 174 not limited to the hippocampal region (Extended Data Fig. 2g).

176 Glycine is released during oxygen-glucose deprivation.

177 Immunohistochemical data illustrated in extended data figure 3 suggest that glycine may be co178 localized in glutamatergic neurons, we therefore hypothesised that depolarization of glutamatergic 179 CA1 pyramidal neurons during the oxygen-glucose deprivation (OGD) paradigm could result in 180 detectable local glycine release ${ }^{29}$. In order to detect glycine release during an OGD paradigm, we 181 used the sniffer-patch technique, wherein activation of glycine receptor $\alpha 2$ subunit indicated 182 glycine release ${ }^{30}$. When the OGD perfusate was applied to the slice, there was a marked increase 183 in the frequency of channel opening in the patch (Extended Data Fig. 4a) and a significant increase 
184 in open probability $\left(P_{\text {open }}\right)$ compared to control (Extended Data Fig. $\left.4 b\right)$. Overall, these results 185 strongly suggest that during OGD conditions, glycine is released into the CA1 extracellular space.

186 Given that multiple studies have demonstrated that glycine receptors (GlyRs) are only weakly 187 expressed at CA1 hippocampal synapses ${ }^{30-32}$, we speculated that the target for the glycine release 188 following OGD could be NMDARs.

\section{OGD paradigm on acute slices, in vitro, decreases NMDAR current amplitude}

191 In brief, we found that an OGD paradigm applied to acute slices during a train stimulation induced 192 NMDAR internalization (Extended Fig. 4c-g). To further confirm that glycine is responsible, we 193 purified glycine oxidase (GO), an enzyme that catalyzes the breakdown of glycine. After 194 demonstrating the effectiveness of purified GO on exogenous glycine levels (Extended Data Fig. $4 \mathrm{~h})$, NMDAR-EPSC trains $(20 \mathrm{~Hz})$ were recorded with $\mathrm{GO}$ and the decrease of the NMDAR-EPSC 196 amplitude was abolished following OGD (Extended Data Fig. 4i-j). Altogether, these in vitro data 197 demonstrate that glycine levels increase during ischemia, however, GINI is only triggered when 198 we further elevate glycine using the train stimulation paradigm. Therefore, we speculated that GINI could also be triggered in vivo during stroke in mice with elevated glycine levels.

\section{Genetic elevation of brain glycine reduces infarct size following photothrombosis}

Glycine has been shown to be neuroprotective in both in vitro ${ }^{31}$ and in vivo models of stroke ${ }^{32-35}$, yet proposed mechanisms have never been expanded into feasible pharmacotherapies. To determine if high glycine levels could result in a decrease in neuronal death following ischemia, we used a well-established focal ischemic paradigm, photothrombosis (PT). Since our in vitro data demonstrate that high glycine/D-serine levels are required to trigger GINI, one would expect that the stroke volume in $\mathrm{GlyT}^{+/-}$mice should be smaller than that observed in WT mice. Indeed, there was a statistically significant decrease in stroke volume in the GlyT1 ${ }^{+/}$mice compared to WT. In contrast, stroke volumes were larger in $\mathrm{SR}^{-/}$mice, compared to WT (Fig. 2a).

211 Pharmacological elevation of brain glycine reduces infarct size following photothrombosis

212 To acutely increase the levels of endogenous glycine, WT mice were treated with NFPS $24 \mathrm{hrs}$ pre213 stroke ${ }^{21-24}$. Forty-eight hours following PT stroke in both the saline- and NFPS-treated cohorts, 214 stroke volume was quantified using 2,3,5-triphenyltetrazolium chloride (TTC) or via magnetic 
215 resonance imaging (MRI). The box-and-whisker plot shows a statistically significant decrease in

216 median stroke volume in the NFPS-treated mice compared to the saline-treated mice (Fig. 2b).

217 This decrease in infarct volume following NFPS treatment is consistent with what has been

218 previously observed in the transient middle cerebral artery occlusion (tMCAO) model of ischemic

219 stroke $^{36,37}$. In addition, FluoroJade C (FJC) staining demonstrated that the NFPS-treated mice also

220 have significantly decreased levels of cell death compared with the saline-treated mice (Extended

221 Data Fig. 5a). Therefore, these data demonstrate that the blockade of GlyT1 is required for the

222 reduction of stroke volume. Interestingly, this decrease in stroke volume was maintained when

223 NFPS was administered up to 10mins post-stroke (Fig. 2d).

Pharmacological elevation of brain glycine minimizes motor behavioural deficits following photothrombosis

Although encouraging, a decrease in stroke volume does not necessarily correlate with a decrease

in post-stroke behavioural deficits ${ }^{38}$. To determine if pre-treatment with NFPS could minimize post-stroke behavioural deficits, we used a well-established behavioural test of motor function, the adhesive removal test ${ }^{39}$. Following PT, a significant attenuation of post-stroke motor behavioural deficits was observed in the cohort of mice treated with NFPS in both time to contact and time to remove, with no significant stroke or drug effect on the unimpaired paw (Fig. 2c).

Pre-stroke administration of NFPS decreases stroke volume and improves motor behavioural deficits following endothelin-1 stroke

The PT stroke model does not recapitulate all of the clinical aspects of ischemia, particularly with respect to reperfusion of the infarct ${ }^{40}$. Therefore, to ensure that the observed decrease in stroke volume and attenuation of behavioural deficits was not an artefact of the PT stroke model, we repeated the experiments using a second known model of focal stroke, the endothelin-1 (ET-1) model $^{37}$. Mice pre-treated with NFPS had significantly smaller ET-1 stroke volumes compared

243 In the adhesive removal task, NFPS-treated mice showed significantly less post-stroke 244 impairments in the impaired paw than the saline-treated mice, in both time to contact and time to 245 remove (Fig. 2f). There was no significant stroke or drug effect on the unimpaired paw 
246 (Supplementary adhesive and cylinder task ${ }^{41}$ data for both stroke models in Extended Data Fig. 247 5b-e and validation of ET-1 model in Extended Data Fig. 6a-c). The horizontal ladder test was an 248 additional assessment of motor function ${ }^{42}$. Following ET-1 stroke, there was a significant increase

249 in impaired paw misses in the saline-treated group, however, in the NFPS-treated group, no 250 significant increase in misses was observed (Extended Data Fig. 5f-g). These data demonstrate 251 that the blockade of GlyT1 ameliorated post-stroke outcomes in two models of stroke. 252 Furthermore, this effect was not due to hypothermia (Extended Data Fig. 6e). Therefore, these data 253 emphasize the crucial role of GlyT1-A in the observed neuroprotection, and this is likely occurring 254 due to GINI.

Blocking NMDAR internalization abolishes the neuroprotective effect of NFPS on stroke volume and behaviour

258 GINI is driven by the recruitment of AP-2 and is mediated by A714 on the C-terminal domain of 259 GluN1. Glycine priming for internalization is specific to A714, therefore this residue is necessary 260 for priming of NMDARs containing either GluN2A or GluN2B in recombinant systems ${ }^{13}$. To 261 confirm that our in vivo observations are due to GINI, we introduced a point mutation into the 262 NMDAR GluN1 subunit (A714L), which abolishes glycine-mediated NMDAR internalization in $263 v$ vitro $^{13}$. We first assessed the functionality of the mutation via transient transfection of GluN1-WT 264 or GluN1-A714L together with WT GluN2A subunit into HEK293 cells resulting in a functional 265 NMDAR. Application of 1mM glycine in cells expressing GluN1-WT induced a significant 266 decrease in the amplitude of the NMDAR-EPSC, while in cells expressing GluN1-A714L this 267 concentration significantly increased the NMDAR amplitude (Extended Data Fig. 7a-b). To 268 visually confirm that GINI was occurring, the movements of NMDARs were tracked over time by 269 live-cell imaging following application of $1 \mathrm{mM}$ glycine (Fig. 3a; Extended Data Fig. 7c; Supplementary Video 1 and 2).

272 This mutation was then packaged into an adeno-associated virus (AAV) 2/9 and injected into the 273 sensory-motor cortex of mice. The functionality of the mutation was re-assessed in acute slices. A 274 dose of $1 \mathrm{mM}$ glycine did not decrease NMDAR-EPSC amplitudes in cells infected with the AAV275 GluN1-A714L constructs (Extended Data Fig. 7d). The spread of the virus occupied a volume that 276 was comparable to the PT stroke (Fig. 3b; Extended Data Fig. 8a), and there were no significant 
277 differences in the PT-induced stroke volume between the mice infected with either the AAV278 GluN1-WT or the AAV-GluN1-A714L constructs (Fig. 3c). However, there was a significant 279 decrease in stroke volume following pre-treatment with NFPS in mice infected with AAV-GluN1280 WT. NFPS administration had no effect on stroke volume in the mice infected with AAV-GluN1281 A714L.

283 The adhesive removal test was repeated on mice infected with either the AAV-GluN1-A714L 284 mutation or the AAV-GluN1-WT. Administration of NFPS to the mice infected with AAV-GluN1285 WT resulted in a significant decrease in post-stroke time to contact and time to remove in the 286 impaired paw (Fig. 3d). Interestingly, in mice infected with the AAV-GluN1-A714L (Fig. 3e), 287 there was no significant change in time to contact and time to remove following stroke in the 288 NFPS-treated mice. Data illustrated in extended data figure $6 \mathrm{~d}$ confirm that the injections of the 289 AAV-GluN1-WT or -GluN1-A714L alone had no effect on behaviour. Taken together, these data 290 confirm that GlyT1-A administration induces neuroprotection in vivo, via GINI.

\section{Pre-stroke administration of NFPS attenuates vascular dysfunction}

293 Stroke is primarily characterized as a vascular disease; therefore, we evaluated the impact of NFPS 294 on vascular function and morphology following PT stroke. Using Laser Doppler flowmetry (LDF), 295 we found that PT stroke induced a significant decrease in blood flow and this effect was rescued with NFPS pre-treatment (Fig. 4a). In Gly $1^{+/-}$mice there was no significant change in blood flow following stroke. Interestingly, in $\mathrm{SR}^{-/}$mice, $\mathrm{PT}$ stroke induced highly significant decrease in blood flow (Fig. 4b).

We next assessed if NFPS could modify vascular morphology by pairing transcardial perfusions of a fluorescent dye with tissue clearing and light sheet fluorescence microscopy (LSFM). This strategy allowed for complete labeling of the cerebral vasculature (Fig. 4c; Supplementary Video 3 and 4). We first used a deep learning segmentation model to automatically calculate stroke volume from our cleared tissue (Fig. 4d-e; Extended Data Fig. 8b;). We observed a decrease in stroke volume in NFPS-treated mice compared to saline-treated mice (Fig. 4e). These results are in agreement with data illustrated in figure $2 \mathrm{~b}$. We further explored the effect of NFPS following PT on vascular density. The PT-induced decrease in vascular density was attenuated with NFPS 
308 treatment compared to saline-treated mice, in the peri-infarct region (Fig. $4 \mathrm{f}-\mathrm{g}$ ). Furthermore, 309 NFPS pre-treatment decreased the PT-induced loss in vessels of smaller diameter and length (Fig. 310 4h-i) compared to saline-treated mice, in the peri-infarct region. Taken together, treatment with

311 NFPS before an ischemic event protects the function and morphology of the cerebral vasculature.

\section{Discussion}

314 Despite evidence from animal studies that NMDAR antagonists minimize neuronal loss ${ }^{43-46}$, 315 clinical trials targeting different binding sites of NMDARs failed due to poor tolerance and lack 316 of efficacy ${ }^{47-49}$. This widespread inhibition of NMDAR function is not compatible with neuronal 317 communication. In this study, we show that GINI is not a direct antagonism of NMDARs but rather 318 a dynamic and reversible phenomenon which dampens NMDAR-mediated excitotoxicity during 319 ischemia while maintaining basal synaptic activity of NMDARs. Several GlyT1-As have been 320 tested and proven to be safe and well tolerated in human clinical trials ${ }^{50}$ and therefore could lead 321 to an innovative clinical approach for the treatment of stroke. 


\section{Main references}

3231 Wu, Q. J. \& Tymianski, M. Targeting NMDA receptors in stroke: new hope in neuroprotection. Mol Brain 11, 15, (2018).

2 Rosenmund, C., Stern-Bach, Y. \& Stevens, C. F. The tetrameric structure of a glutamate receptor channel. Science 280, 1596-1599, (1998).

3273 Johnson, J. W. \& Ascher, P. Glycine potentiates the NMDA response in cultured mouse brain neurons. Nature 325, 529-531, (1987).

3294 Kleckner, N. W. \& Dingledine, R. Requirement for glycine in activation of NMDAreceptors expressed in Xenopus oocytes. Science 241, 835-837, (1988). Nong, Y. et al. Glycine binding primes NMDA receptor internalization. Nature 422, 302$5 \quad$ Nong, Y. et al.

6 Nong, Y., Huang, Y. Q. \& Salter, M. W. NMDA receptors are movin' in. Curr Opin Neurobiol 14, 353-361, (2004).

7 Aragon, M. C., Gimenez, C. \& Mayor, F. Stoichiometry of sodium- and chloride-coupled glycine transport in synaptic plasma membrane vesicles derived from rat brain. FEBS Lett 212, 87-90, (1987).

8 Guastella, J., Brecha, N., Weigmann, C., Lester, H. A. \& Davidson, N. Cloning, expression, and localization of a rat brain high-affinity glycine transporter. Proc Natl Acad Sci US A 89, 7189-7193, (1992).

9 Smith, K. E., Borden, L. A., Hartig, P. R., Branchek, T. \& Weinshank, R. L. Cloning and expression of a glycine transporter reveal colocalization with NMDA receptors. Neuron $\mathbf{8}$, 927-935, (1992).

10 Bergeron, R., Meyer, T. M., Coyle, J. T. \& Greene, R. W. Modulation of N-methyl-Daspartate receptor function by glycine transport. Proc Natl Acad Sci U S A 95, 1573015734, (1998).

11 Forsythe, I. D., Westbrook, G. L. \& Mayer, M. L. Modulation of excitatory synaptic transmission by glycine and zinc in cultures of mouse hippocampal neurons. J Neurosci $\mathbf{8}$, 3733-3741, (1988).

12 Paoletti, P., Neyton, J. \& Ascher, P. Glycine-independent and subunit-specific

13 Han, L., Campanucci, V. A., Cooke, J. \& Salter, M. W. Identification of a single amino acid in GluN1 that is critical for glycine-primed internalization of NMDA receptors. $\mathrm{Mol}$ Brain 6, 36, (2013).

14 Kirchhausen, T., Macia, E. \& Pelish, H. E. Use of dynasore, the small molecule inhibitor of dynamin, in the regulation of endocytosis. Methods Enzymol 438, 77-93, (2008).

15 Lai, M. M. et al. The calcineurin-dynamin 1 complex as a calcium sensor for synaptic vesicle endocytosis. J Biol Chem 274, 25963-25966, (1999). Traynelis, S. F. et al. Glutamate receptor ion channels: structure, regulation, and function. Pharmacol Rev 62, 405-496, (2010).

17 Gomeza, J. et al. Inactivation of the glycine transporter 1 gene discloses vital role of glial glycine uptake in glycinergic inhibition. Neuron 40, 785-796, (2003).

18 Tsai, G. et al. Gene knockout of glycine transporter 1: characterization of the behavioral phenotype. Proc Natl Acad Sci U S A 101, 8485-8490, (2004).

19 Papouin, T. et al. Synaptic and extrasynaptic NMDA receptors are gated by different endogenous coagonists. Cell 150, 633-646, (2012). 
20 Wolosker, H. et al. Purification of serine racemase: biosynthesis of the neuromodulator Dserine. Proc Natl Acad Sci U S A 96, 721-725, (1999). Aubrey,
K. R. \&
Vandenberg, R. J.
J. $\quad \mathrm{N}[3-(4$ '-fluorophenyl)-3-(4'phenylphenoxy)propyl]sarcosine (NFPS) is a selective persistent inhibitor of glycine transport. Br J Pharmacol 134, 1429-1436, (2001).

22 Herdon, H. J. et al. Pharmacological assessment of the role of the glycine transporter GlyT1 in mediating high-affinity glycine uptake by rat cerebral cortex and cerebellum synaptosomes. Neuropharmacology 41, 88-96, (2001).

23 Mallorga, P. J. et al. Pharmacology and expression analysis of glycine transporter GlyT1 with [3H]-(N-[3-(4'-fluorophenyl)-3-(4'phenylphenoxy)propyl])sarcosine. Neuropharmacology 45, 585-593, (2003).

24 Liu, X. et al. Use of a physiologically based pharmacokinetic model to study the time to reach brain equilibrium: an experimental analysis of the role of blood-brain barrier permeability, plasma protein binding, and brain tissue binding. J Pharmacol Exp Ther 313, 1254-1262, (2005).

25 Pinto, M. C. et al. Glycine transporters type 1 inhibitor promotes brain preconditioning against NMDA-induced excitotoxicity. Neuropharmacology 89, 274-281, (2015).

26 Basu, A. C. et al. Targeted disruption of serine racemase affects glutamatergic neurotransmission and behavior. Mol Psychiatry 14, 719-727, (2009).

27 Balu, D. T., Basu, A. C., Corradi, J. P., Cacace, A. M. \& Coyle, J. T. The NMDA receptor co-agonists, D-serine and glycine, regulate neuronal dendritic architecture in the somatosensory cortex. Neurobiol Dis 45, 671-682, (2012).

28 Benneyworth, M. A. \& Coyle, J. T. Altered acquisition and extinction of amphetaminepaired context conditioning in genetic mouse models of altered NMDA receptor function. Neuropsychopharmacology 37, 2496-2504, (2012).

29 Rossi, D. J., Brady, J. D. \& Mohr, C. Astrocyte metabolism and signaling during brain ischemia. Nat Neurosci 10, 1377-1386, (2007).

30 Muller, E. et al. Vesicular storage of glycine in glutamatergic terminals in mouse hippocampus. Neuroscience 242, 110-127, (2013).

$31 \mathrm{Hu}, \mathrm{R}$. et al. Glycine triggers a non-ionotropic activity of GluN2A-containing NMDA receptors to confer neuroprotection. Sci Rep 6, 34459, (2016).

32 Chen, Z. et al. Glycine bidirectionally regulates ischemic tolerance via different mechanisms including NR2A-dependent CREB phosphorylation. J Neurochem 133, 397 408, (2015).

33 Chen, J. et al. A non-ionotropic activity of NMDA receptors contributes to glycine-induced neuroprotection in cerebral ischemia-reperfusion injury. Sci Rep 7, 3575, (2017).

34 Zhao, D. et al. Glycine confers neuroprotection through PTEN/AKT signal pathway in experimental intracerebral hemorrhage. Biochem Biophys Res Commun 501, 85-91, (2018).

35 Qin, X. et al. MicroRNA-26b/PTEN Signaling Pathway Mediates Glycine-Induced Neuroprotection in SAH Injury. Neurochem Res 44, 2658-2669, (2019).

36 Huang, B. et al. GlyT1 Inhibitor NFPS Exerts Neuroprotection via GlyR Alpha1 Subunit in the Rat Model of Transient Focal Cerebral Ischaemia and Reperfusion. Cell Physiol Biochem 38, 1952-1962, (2016).

37 Dojo Soeandy, C. et al. Endothelin-1-mediated cerebral ischemia in mice: early cellular events and the role of caspase-3. Apoptosis 24, 578-595, (2019). 
41338 Pineiro, R. et al. Relating MRI changes to motor deficit after ischemic stroke by segmentation of functional motor pathways. Stroke 31, 672-679, (2000).

39 Bouet, V. et al. The adhesive removal test: a sensitive method to assess sensorimotor deficits in mice. Nat Protoc 4, 1560-1564, (2009).

40 Sommer, C. J. Ischemic stroke: experimental models and reality. Acta Neuropathol 133, 245-261, (2017).

$41941 \quad$ Schallert, T., Fleming, S. M., Leasure, J. L., Tillerson, J. L. \& Bland, S. T. CNS plasticity and assessment of forelimb sensorimotor outcome in unilateral rat models of stroke, cortical ablation, parkinsonism and spinal cord injury. Neuropharmacology 39, 777-787, (2000).

42 Metz, G. A. \& Whishaw, I. Q. The ladder rung walking task: a scoring system and its practical application. J Vis Exp, (2009).

43 Gotti, B. et al. Ifenprodil and SL 82.0715 as cerebral anti-ischemic agents. I. Evidence for efficacy in models of focal cerebral ischemia. J Pharmacol Exp Ther 247, 1211-1221, (1988).

44 Park, C. K., Nehls, D. G., Teasdale, G. M. \& McCulloch, J. Effect of the NMDA antagonist MK-801 on local cerebral blood flow in focal cerebral ischaemia in the rat. $J$ Cereb Blood Flow Metab 9, 617-622, (1989).

45 Scatton, B. Excitatory amino acid receptor antagonists: a novel treatment for ischemic cerebrovascular diseases. Life Sci 55, 2115-2124, (1994).

46 Prass, K. \& Dirnagl, U. Glutamate antagonists in therapy of stroke. Restor Neurol Neurosci 13, 3-10, (1998).

47 Ikonomidou, C. \& Turski, L. Why did NMDA receptor antagonists fail clinical trials for stroke and traumatic brain injury? Lancet Neurol 1, 383-386, (2002).

48 Lipton, S. A. Failures and successes of NMDA receptor antagonists: molecular basis for the use of open-channel blockers like memantine in the treatment of acute and chronic neurologic insults. NeuroRx 1, 101-110, (2004).

49 Kalia, L. V., Kalia, S. K. \& Salter, M. W. NMDA receptors in clinical neurology: excitatory times ahead. Lancet Neurol 7, 742-755, (2008).

50 Harvey, R. J. \& Yee, B. K. Glycine transporters as novel therapeutic targets in schizophrenia, alcohol dependence and pain. Nat Rev Drug Discov 12, 866-885, (2013). 


\section{Animals}

All procedures in this study were carried out on female and/or male 8-10-week-old mice in accordance with the guidelines of the Canadian Council on Animal Care and approved by the

451 University of Ottawa Animal Care Committee. The following transgenic mouse lines were 452 utilized: heterozygous glycine transporter type $1\left(\mathrm{GlyT}^{+/-}\right)$, serine racemase knockout $\left(\mathrm{SR}^{-/-}\right)$, 453 and $N$-methyl-D-aspartate receptor (NMDAR) GluN2A subunit knockout (GluN2A ${ }^{-/-}$) mice ${ }^{51}$, 454 along with their wild type (WT) litter mates (on $\mathrm{C} 57 \mathrm{Bl} / 6 ; \mathrm{S} 129, \mathrm{C} 57 \mathrm{Bl} / 6 ; \mathrm{S} 129$ and $\mathrm{C} 57 \mathrm{Bl} / 6$ 455 backgrounds respectively). In vivo behavioural experiments were performed on C57Bl/6 WT mice 456 from Charles River ${ }^{\circledR}$. The animals were housed under standard conditions and had access to chow 457 and water ad libitum.

\section{Whole-cell electrophysiology on hippocampal slices and HEK293 cells}

460 Whole-cell voltage-clamp recordings were obtained from visually identified CA1 pyramidal cells 461 from acute hippocampal brain slices $(300 \mu \mathrm{m}$ thick) in oxygenated artificial cerebrospinal fluid 462 (ACSF) as previously described ${ }^{52,53}$. The cells were voltage-clamped at $-65 \mathrm{mV}$ using cesium 463 methane sulfonate based internal solution and postsynaptic currents were evoked by electrical 464 stimulation of the Schaffer collaterals with a bipolar stimulating electrode positioned in the stratum radiatum. The intensity of the stimulation was adjusted to obtain evoked excitatory postsynaptic currents (EPSCs). The stimulation protocol consisted of a single $100 \mu$ s current pulse (10-200 $\mu \mathrm{A})$

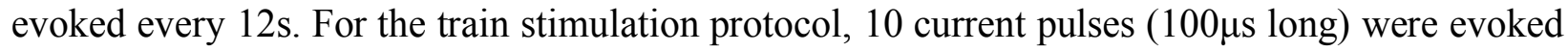
at $50 \mathrm{~Hz}$ for $200 \mathrm{~ms}$ and then repeated once every $20 \mathrm{~s}$. To isolate the NMDAR-EPSC, a low concentration of $\mathrm{MgCl}_{2}(0.13 \mathrm{mM})$ ACSF was used wherein the $\mathrm{CaCl}_{2}$ concentration was increased to $3.5 \mathrm{mM}$ to maintain cation balance.

472 HEK293 cells were used for electrophysiology recordings 48-72hrs following transfection with 473 either the GluN1-WT or GluN1-A714L cDNA along with an equimolar ratio of the fluorescent 474 marker cDNA, GFP-GluN2A. NMDAR currents were evoked using pressure ejection (10psi) from 475 a picospritzer micropipette filled with $10 \mu \mathrm{M}$ glycine and $1 \mathrm{mM}$ glutamate (Sigma-Aldrich) for a 476 duration of $25-50 \mathrm{~ms}$ every $20 \mathrm{~s}$ at a membrane potential of $-60 \mathrm{mV}$. HEK293 cells were recorded 
477 in HEPES-buffered saline external solution with low $\mathrm{MgCl}_{2}$ using a potassium gluconate recording 478 solution.

480 When required, additional drugs were applied including various concentrations of D-serine and 481 glycine (Millipore Sigma), as well as 300nM $N$-[3-(4'-fluorophenyl)-3-(4'482 phenylphenoxy)propyl]sarcosine (NFPS; Tocris Bioscience), 10mM BAPTA (Thermo Fisher), $48330 \mu \mathrm{M}$ cyclopiazonic acid (CPA) (Tocris Bioscience), and 20 $\mu \mathrm{M}$ nimodipine (Millipore Sigma). 484 The inhibitors of clathrin-mediated endocytosis, $100 \mu \mathrm{M}$ dynasore (Millipore Sigma), $100 \mu \mathrm{M}$ 485 dynamin blocking peptide (DBP; Tocris Bioscience), and 30 $\mu \mathrm{M}$ Dyngo4a (Abcam) were included 486 in the internal solution.

\section{Sniffer-patch technique}

489 To detect glycine release, we used the "sniffer patch" technique ${ }^{54-57}$. A Chinese Hamster Ovary 490 (CHO) cell line were generated and stably transfected with the $\alpha 2$ subunit of the glycine receptor $491(\mathrm{GlyR})^{58}$. Outside-out membrane patches were excised from the CHO cells using thick-walled 492 borosilicate glass pipettes filled with a cesium chloride internal solution. Following patch excision, 493 the electrode was placed in the stratum radiatum of the CA1 region of the hippocampus to detect 494 glycine release and allow channel activation. Channel open probability $\left(P_{\text {open }}\right)$ was derived by 495 measuring the mean open time of all the single channel events during the recording window, then

496 dividing by the sum of the mean open and shut times. Multiple channel openings were set as a $497 \quad P_{\text {open }}=1$ for that particular time period.

\section{Oxygen-glucose deprivation paradigm}

500 To mimic ischemia, the acute slices were challenged by an oxygen-glucose deprivation paradigm

501 (OGD) modified from Rossi et al. ${ }^{59}$. In this paradigm, external glucose was replaced with $7 \mathrm{mM}$ 502 sucrose, and the external solution was saturated with $95 \% \mathrm{~N}_{2} / 5 \% \mathrm{CO}_{2}$ instead of $95 \% \mathrm{O}_{2} / 5 \%$ $503 \mathrm{CO}_{2}$. Iodoacetate and cyanide were also added to the OGD external solution to block glycolysis 504 and oxidative phosphorylation.

\section{Purification of glycine oxidase}


507 The plasmid containing His-tagged glycine oxidase (GO) was generated from Bacillus subtilis.

508 This plasmid was a gift from Dr. Steven Ealick (Cornell University, NY, USA). The protein was 509 expressed in E. coli and purified as previously described ${ }^{60-64}$.

\section{NMDAR internalization imaging in HEK293 cells}

512 HEK293 cells were transiently transfected with either GluN1-WT or GluN1-A714L cDNAs

513 together with pHluorin-GluN2A cDNA. HEK293 cells were then grown for 24-48hrs in the 514 presence of D-APV (Tocris Bioscience). Images were acquired with an LSM880 Confocal 515 Microscope (Zeiss), with cells in a modified HEPES buffer (add 1mM Glutamate, omit $0.13 \mathrm{mM}$ $516 \mathrm{MgCl}_{2}$ ). A 647nm-tagged FluoTag ${ }^{\circledR}-\mathrm{X} 4$ anti-GFP (1:250-1:500; NanoTag Biotechnologies) was

517 added to tag extracellular NMDARs prior to the acute application of an internalizing dose of 518 glycine. The nanotags (anti-GFP nanobody) are cell impermeable and tag NMDARs on the cell 519 surface. Therefore, the nanotags were observed within the cell only when NMDARS had been 520 internalized. Images were acquired every 3 mins over $10-12$ mins to visualize internalization. 521 Internalization was deemed to have occurred when the cell-impermeable NanoTag (647) was 522 observed within the cell.

\section{Immunohistochemistry}

525 Slices were washed then permeabilized with $0.25 \%(\mathrm{w} / \mathrm{v})$ gelatin and $0.2 \%$ Triton X-100 (v/v) in 526 PBS (PBS-GT). Slices were incubated in primary antibodies in PBS-GT overnight, then washed 527 three times with PBS-GT before the addition of fluorescent secondary antibodies at room 528 temperature (RT). Slices were rinsed, air-dried, and mounted onto slides (Extended Data Table $5291)^{65}$.

531 The channels representing glycine $(650 \mathrm{~nm})$, vesicular glutamate transporter (VGLUTs 1-2; $532488 \mathrm{~nm}$ ), and vesicular GABA transporter (VGAT;550nm) were merged into a single image using 533 Adobe Photoshop 7.01 and all quantifications were done manually by a researcher blinded to the 534 treatment groups. Platelet endothelial cell adhesion molecule-1 (CD-31; 647nm) and Collagen IV 535 (CollIV;647nm) were depicted in purple and visually colocalized to filled, perfused vessels $536(488 \mathrm{~nm})$. 


\section{Generation of WT and A714L viral constructs}

539 The GluN1 constructs were made by cloning the GluN1 coding region of a SuperEcliptic Phluorin (SEP)-tagged GluN1 construct (Addgene \#23999) into pDrive cloning vector (pDrive cloning vector, Qiagen). We then used this as a template to create the A714L mutant clone by site-directed

542 mutagenesis. These cDNAs were used for transfection of HEK293 cells. For generation of GluN1-

543 WT and GluN1-A714L adeno-associated virus (AAV), the coding fragments of these

544 constructs were sub-cloned into an adeno-associated viral vector, and viral constructs were then

545 packaged with plasmid AAV2/9 at the University of Laval.

\section{Photothrombosis and Endothelin-1 Stroke}

548 NFPS or a vehicle control solution was injected intraperitoneally (i.p.) into C57Bl/6 mice either $54924 \mathrm{hrs}$ prior to stroke or $10 \mathrm{mins} / 60 \mathrm{mins} / 120 \mathrm{mins}$ post-stroke, at a dose of $5 \mathrm{mg} / \mathrm{kg}$. 550 Photothrombotic $(\mathrm{PT})^{66}$ or cortical endothelin-1 (ET-1) ${ }^{67}$ strokes were induced as previously 551 described. Mice were anesthetized with 2.5\% isoflurane in $\mathrm{O}_{2}$ and mounted onto a stereotaxic 552 frame. For PT strokes, a dose of $10 \mathrm{mg} / \mathrm{mL}$ of Rose Bengal (Tocris) was injected i.p.. Immediately 553 following the injection of the dye, the skull was exposed to visualize bregma. Using the stereotaxic 554 device, a 520nm laser ( 20mW; Beta Electronics) was positioned above the sensorimotor cortex $555(\mathrm{AP}+0.7, \mathrm{ML}+2.0)$ and turned on for $10 \mathrm{mins}$ to induce a permanent occlusion. For ET-1 strokes, 556 once the skull was exposed and a craniotomy performed for each injection site (1. AP $+0.0, \mathrm{ML}$ $557+2.0, \mathrm{DV}-1.6 ; 2$. AP +0.2, $\mathrm{ML}+2.0, \mathrm{DV}-1.4 ; 3$. AP $+0.4, \mathrm{ML}+2.0, \mathrm{DV}-1.3), 1 \mu \mathrm{L}$ of $2 \mu \mathrm{g} / \mu \mathrm{L}$ 558 human, porcine ET-1 (Abcam), dissolved in $2.7 \mu \mathrm{g} / \mu \mathrm{L}$ L-NAME (Abcam), was injected over $5595 \mathrm{mins}$ with a $28 \mathrm{G} 10 \mu \mathrm{L}$ Hamilton syringe to induce a transient ischemic stroke.

\section{Cortical infection with AAVs}

562 Mice were anesthetized with 2.5\% isoflurane in $\mathrm{O}_{2}$ and mounted onto a stereotaxic apparatus. The 563 intact skull was exposed to visualize bregma. The following injection sites were measured from 564 bregma: 1. AP +1.2, ML +2.0, DV -0.5; 2. AP +0.2, ML +2.0, DV -0.5. A craniotomy was 565 performed at each site prior to injecting $0.5 \mu \mathrm{L}$ of $10^{-12} \mathrm{PFU} / \mathrm{mL}$ (plaque forming units) of either a 566 AAV-WT-GluN1 or the mutant AAV-GluN1-A714L construct, over 5mins with a $28 \mathrm{G} 10 \mu \mathrm{L}$ 567 Hamilton syringe. 
Laser Doppler Flowmetry (LDF) recordings following PT were performed as previously

571 described $^{68}$. Mice were anesthetized with an i.p. injection of a $0.01 \mathrm{ml} / \mathrm{g}$ cocktail consisting of

$572120 \mathrm{mg} / \mathrm{kg}$ ketamine and $10 \mathrm{mg} / \mathrm{kg}$ xylazine, and then mounted onto a stereotaxic apparatus.

573 Following exposure and thinning of the skull, the laser probe (Transonic Systems) was positioned

574 over the sensory motor cortex ( $\mathrm{AP}+0.7, \mathrm{ML}+2.0)$ and baseline activity was recorded for 5 mins.

575 The laser probe was replaced with a $520 \mathrm{~nm}$ laser ( 20mW; Beta Electronics) to induce PT stroke,

576 as described above. Following PT, LDF recordings were performed for an additional 30mins.

Quantification of stroke volume - Magnetic resonance imaging:

579 Magnetic resonance imaging (MRI) was performed at the University of Ottawa pre-clinical imaging core using a 7 Tesla GE/Agilent MR 901. Mice were anaesthetized for the MRI procedure using isoflurane in $\mathrm{O}_{2}$ : induction at 3\%, maintenance at 1.5\% . A 2D fast spin echo sequence (FSE) pulse sequence was used for the imaging, with the following parameters: slice thickness $=0.5 \mathrm{~mm}$, spacing $=0 \mathrm{~mm}$, field of view $=2.5 \mathrm{~cm}$, matrix $=256 \times 256$, echo time $=41 \mathrm{~ms}$, repetition time $=$ $7000 \mathrm{~ms}$, echo train length $=8$, bandwidth $=16 \mathrm{kHz}$, fat saturation. Stroke lesions demonstrated hyperintensity.

Quantification of stroke volume - Triphenyltetrazolium chloride:

588 Stroke volume quantification was performed using 2,3,5-triphenyltetrazolium chloride (TTC; 589 Sigma $)^{69,70}$. Forty-eight hours post-stroke, mice were deeply anesthetized with $5 \%$ isoflurane in $590 \mathrm{O}_{2}$ before decapitation for slicing on a vibratome (Leica) in cold ACSF at $0.5 \mathrm{~mm}$. Slices were 591 incubated in $2 \% \mathrm{TTC}$ at $37^{\circ} \mathrm{C}$ for $10 \mathrm{mins}$, then transferred to $4 \%$ paraformaldehyde (PFA) at $4{ }^{\circ} \mathrm{C}$.

592 Brain slices were imaged from both sides and the surface area of the infarct regions were measured on Fiji (ImageJ.com) and multiplied by the thickness of the slice to obtain a final volume.

\section{Quantification of stroke volume - Cresyl violet:}

596 Forty-eight hours post-stroke, mice were transcardially perfused with 1X PBS, followed by 4\% 597 PFA. Brains were collected and post-fixed in 4\% PFA overnight and then incubated in sucrose 598 until saturation. Serial $25 \mu \mathrm{m}$ thick coronal sections were cut on a cryostat (Microm HM500), and 
600 were immersed in xylene and then rehydrated in decreasing concentrations of ethanol before being 601 placed in double distilled water $\left(\mathrm{ddH}_{2} \mathrm{O}\right)$. Once rehydrated, slides were stained with cresyl violet 602 (CV) (Electron Microscopy Sciences) and placed in $\mathrm{dd}_{2} \mathrm{O}$. Slides were then dehydrated in 603 increasing concentrations of ethanol before being immersed in xylene. Once removed, the slides 604 were mounted with DPX mounting media (Sigma). Images of CV-stained slices were acquired with the EVOS FLAuto2 inverted epifluorescence microscope under brightfield. The surface area of the infarct regions was multiplied by the distance between each collected slice $(500 \mu \mathrm{m})$ to obtain a volume. The sum of all slices was used to obtain a final stroke volume per brain.

\section{Quantification of neuronal loss - FluoroJade C:}

610 The brain tissue preparation for FluoroJade C (FJC; EMD Millipore) was treated exactly as that of 611 the CV brain tissue. Slides were first immersed in 1\% sodium hydroxide in $80 \%$ ethanol, $70 \%$ 612 ethanol and finally in $\mathrm{ddH}_{2} \mathrm{O}$ before being incubated in a $0.06 \%$ potassium permanganate (Sigma613 Aldrich) solution. This was followed by an incubation in a $0.0001 \%$ FJC solution dissolved in $6140.1 \%$ aqueous acetic acid and combined with 0.0001\% DAPI (Santa Cruz Biotechnology), and 615 slides were once again rinsed with $\mathrm{ddH}_{2} \mathrm{O}$ and left to air dry. Slides were then immersed in xylene 616 and mounted with FluoroMountG (Sigma-Aldrich) ${ }^{71}$. Imaging was completed with the Zeiss 617 AxioObserver Z1 inverted epifluorescence microscope using GFP (488/509nm) and DAPI $618(359 / 461 \mathrm{~nm})$ filters. Analysis of the total number of degenerating neurons was performed using 619 IMARIS 9.2 (Bitplane). IMARIS 9.2 was set to detect and count all green (representing 620 degenerating neurons) and blue (representing nuclear DNA) spots on each image and then 621 calculate colocalization. The cells having been tagged by both DAPI and FJC were counted as FJC 622 positive neurons. Tissue clearing

625 CUBIC tissue clearing was completed as previously described ${ }^{72}$. Following perfusion, the tissue 626 was post-fixed overnight in 4\% PFA, then washed in 1X PBS the following day. Following 627 washes, tissue was submerged in half diluted CUBIC-L (1:1, CUBIC-L:Water) at $37^{\circ} \mathrm{C}$ overnight. 628 Tissue was submerged in CUBIC-L at $37^{\circ} \mathrm{C}$ with gentle shaking over 10 days, changing the 629 solution every 48hrs. Tissue was then washed in 1X PBS before being submerged in half diluted 
631 submerged in CUBIC- $\mathrm{R}^{+}(\mathrm{M})$ the following day, then replaced with fresh CUBIC-R $(\mathrm{M}) 24 \mathrm{hrs}$

632 later. Tissue was imaged with a light sheet microscope in a refractive index matched imaging

633 solution consisting of a mixture of HIVAC-4 with mineral oil.

\section{Quantification of stroke volume:}

636 For CUBIC-cleared brains imaged by light sheet fluorescence microscopy (LSFM), infarct volume 637 was measured using a deep learning segmentation model. For more information please refer to the 638 "Light sheet fluorescence microscopy" section below.

\section{Post-stroke vascular morphology quantification:}

641 For analysis of vascular morphology in the peri-infarct region, transcardial FITC-BSA staining 642 was paired with CUBIC brain clearing to allow for LSFM imaging. Forty-eight hours post PT 643 stroke, mice were transcardially perfused with $20 \mathrm{~mL} 1$ X PBS, then $20 \mathrm{~mL} 4 \%$ PFA. Mice were 644 then submerged in a $37^{\circ} \mathrm{C}$ water bath, facing down at an angle of $30^{\circ}$ before being perfused with $64510 \mathrm{~mL}$ of $0.5 \%$ FITC-BSA (Sigma-Aldrich), in 2\% gelatin (Sigma-Aldrich). Subsequently, mice 646 were submerged in an ice bath for $30 \mathrm{mins}$ before the brain was dissected out $^{73}$. Brains were 647 collected and post-fixed in 4\% PFA overnight, then cleared following the CUBIC tissue clearing 648 protocol. Following tissue clearing, brains were imaged by LSFM. A $1.125 \mathrm{~mm}^{3}$ region of interest 649 lateral to the stroke site was manually selected, then analyzed using AIVIA 9 (DRVISION 650 Technologies).

652 Viral spread quantification:

653 Three weeks following cortical infection, mice were transcardially perfused with 1X PBS, 654 followed by $4 \%$ PFA. Brains were sliced into $0.5 \mathrm{~mm}$ coronal slices and cleared following the 655 SeeDB tissue clearing protocol described by Ke et al. ${ }^{74}$. Following tissue clearing, brains were 656 imaged with the Zeiss LSM800 confocal microscope. All viral spread analysis was performed 657 manually using Fiji. 
661 Imaging was performed using our custom-built light sheet microscope. CUBIC-cleared brains 662 were imaged using a 2.5X objective (NA0.07), 488nm excitation laser line and $5 \mu \mathrm{m}$ steps. Each

663 sample was scanned as a series of tiles, then stitched into a single image using TeraStitcher. The 664 stitched scans were then run through AIVIA 9 to segment and create 3D reconstructions of the 665 vascular network. Properties of each vessel (diameter and length) were automatically calculated 666 by AIVIA 9, and then exported for analysis.

\section{Automated stroke volume measurement (stroke volume prediction):}

669 Stroke volume in cleared tissue was calculated as follows: the areas representing stroke in each 670 slice were identified, multiplied by their z-depth (thickness), then summed to obtain a total volume. 671 The stroke regions were identified in each slice using a deep convolutional neural network ${ }^{75-77}$ 672 which was deemed $98 \%$ accurate. The network was first pre-trained on a large data-set ${ }^{78}$, then 673 further trained using 906 experimental scans.

\section{Behavioural tests}

\section{Adhesive removal test:}

677 The adhesive removal test was performed as described ${ }^{79}$. Mice were trained pre-stroke daily over 6785 days and tested post stroke over 2 days. Trials began with $1 \mathrm{~min}$ of habituation to an empty home 679 cage, before strips of adhesive were placed onto both forepaws. The mouse was then placed back 680 into the cage and the times to contact and remove the adhesive strips were recorded by two 681 experimenters. Mice were allotted a maximum of $2 \mathrm{mins}$ to complete the task. The times to contact 682 and remove the pieces of adhesive tapes were compared per paw as well as pre- and post-stroke.

\section{Horizontal ladder test:}

685 The horizontal ladder test was performed based on protocols described previously ${ }^{80,81}$ with slight 686 modifications. Mice underwent one day of training prior to stroke. In the pre-stroke trials, mice 687 crossed the ladder up to seven times or until they had performed two acceptable runs. In turn, 688 during the post-stroke trials, mice had three attempts to cross the ladder, two of which were scored. 689 Each trial was recorded with a video camera. Scoring and analysis were performed by an 690 experimenter blind to the conditions. The video recordings of the best two trials from each mouse 691 were analyzed frame-by-frame with Noldus Observer XT program. Each limb’s step was scored 
692 as either "correct", "partial" or "miss". The percentage of missed steps pre- and post-stroke were 693 compared.

\section{Cylinder test:}

696 The cylinder test was performed based on protocols described ${ }^{82,83}$, with slight modifications. Mice

697 were placed in a transparent cylinder and filmed with an overhead camera until they reared 22 698 times. With each rear, three types of behaviours were recorded: (A) right paw is exclusively weight 699 bearing; (B) left paw is exclusively weight bearing; (C) both paws are weight bearing at the same 700 time. The video recordings of each trial were analyzed frame-by-frame with Noldus Observer XT 701 program by a single experimenter blind to the conditions. The length and frequency of forelimb 702 contacts to the wall of the cylinder were scored. The behaviours were expressed per paw as an 703 average time in relation to the sum of the independent left and right weight bearing. The average 704 time spent on the impaired paw (right) was compared in pre-stroke and post-stroke trials.

\section{Quantification and Statistical analysis}

707 All data are presented as means \pm S.E.M.; $n$ represents the number of mice or cells in each group, 708 as indicated in each figure. In most cases, statistical significance was determined by a paired, two709 tailed Student's t-test or a two-way repeated measure ANOVA followed by Bonferroni post hoc 710 comparisons, for multiple group comparisons. In cases in which datasets had multiple missing 711 values, a mixed-model (ANOVA) was implemented by GraphPad Prism. For analyses of groups 712 across multiple time points, a one-way ANOVA was utilized. Statistical analyses and data 713 presentation were completed using both OriginPro 8.5 (OriginLab Software) and GraphPad Prism 7148 (GraphPad Software).

716 For electrophysiological data, decay kinetic and amplitude analysis were performed on averaged

717 traces. Decay time constants were best fit with a double exponential function and expressed as a 718 weighted mean. Due to summation, EPSC amplitudes in the train were measured from the end of 719 the previous EPSC rather than from the initial baseline.

721 Please refer to Supplementary Methods for more detailed description of all methods. 
72451 Kannangara, T. S. et al. GluN2A-/- Mice Lack Bidirectional Synaptic Plasticity in the

52 Martina, M. et al. Glycine transporter type 1 blockade changes NMDA receptor-mediated responses and LTP in hippocampal CA1 pyramidal cells by altering extracellular glycine levels. J Physiol 557, 489-500, (2004).

53 Martina, M. et al. Reduced glycine transporter type 1 expression leads to major changes in glutamatergic neurotransmission of CA1 hippocampal neurones in mice. J Physiol 563, 777-793, (2005).

54 Allen, T. G. The 'sniffer-patch' technique for detection of neurotransmitter release. Trends Neurosci 20, 192-197, (1997).

55 Lee, C. J. et al. Astrocytic control of synaptic NMDA receptors. J Physiol 581, 1057-1081, (2007).

56 Aubrey, K. R. et al. The transporters GlyT2 and VIAAT cooperate to determine the vesicular glycinergic phenotype. J Neurosci 27, 6273-6281, (2007).

57 Scain, A. L. et al. Glycine release from radial cells modulates the spontaneous activity and its propagation during early spinal cord development. J Neurosci 30, 390-403, (2010).

58 Mangin, J. M. et al. Kinetic properties of the alpha2 homo-oligomeric glycine receptor impairs a proper synaptic functioning. J Physiol 553, 369-386, (2003).

59 Rossi, D. J., Oshima, T. \& Attwell, D. Glutamate release in severe brain ischaemia is mainly by reversed uptake. Nature 403, 316-321, (2000).

60 Job, V., Marcone, G. L., Pilone, M. S. \& Pollegioni, L. Glycine oxidase from Bacillus subtilis. Characterization of a new flavoprotein. J Biol Chem 277, 6985-6993, (2002).

61 Settembre, E. C. et al. Structural and mechanistic studies on ThiO, a glycine oxidase essential for thiamin biosynthesis in Bacillus subtilis. Biochemistry 42, 2971-2981, (2003).

62 Molla, G., Motteran, L., Job, V., Pilone, M. S. \& Pollegioni, L. Kinetic mechanisms of glycine oxidase from Bacillus subtilis. Eur J Biochem 270, 1474-1482, (2003).

63 Pedotti, M., Ghisla, S., Motteran, L., Molla, G. \& Pollegioni, L. Catalytic and redox properties of glycine oxidase from Bacillus subtilis. Biochimie 91, 604-612, (2009).

64 Caldinelli, L., Pedotti, M., Motteran, L., Molla, G. \& Pollegioni, L. FAD binding in glycine oxidase from Bacillus subtilis. Biochimie 91, 1499-1508, (2009).

65 Muller, E. et al. Vesicular storage of glycine in glutamatergic terminals in mouse hippocampus. Neuroscience 242, 110-127, (2013).

66 Lee, J. K. et al. Photochemically induced cerebral ischemia in a mouse model. Surg Neurol 67, 620-625; discussion 625, (2007).

67 Wang, Y., Jin, K. \& Greenberg, D. A. Neurogenesis associated with endothelin-induced cortical infarction in the mouse. Brain Res 1167, 118-122, (2007).

68 Toussay, X., Tiberi, M. \& Lacoste, B. Laser Doppler Flowmetry to Study the Regulation of Cerebral Blood Flow by G Protein-Coupled Receptors in Rodents. Methods Mol Biol 1947, 377-387, (2019).

69 Hatfield, R. H., Mendelow, A. D., Perry, R. H., Alvarez, L. M. \& Modha, P. Triphenyltetrazolium chloride (TTC) as a marker for ischaemic changes in rat brain following permanent middle cerebral artery occlusion. Neuropathol Appl Neurobiol 17, 61-67, (1991). 
Benedek, A. et al. Use of TTC staining for the evaluation of tissue injury in the early phases of reperfusion after focal cerebral ischemia in rats. Brain Res 1116, 159-165, (2006). Ehara, A. \& Ueda, S. Application of Fluoro-Jade C in acute and chronic neurodegeneration models: utilities and staining differences. Acta Histochem Cytochem 42, 171-179, (2009). Matsumoto, K. et al. Advanced CUBIC tissue clearing for whole-organ cell profiling. Nat Protoc 14, 3506-3537, (2019).

Tsai, P. S. et al. Correlations of neuronal and microvascular densities in murine cortex revealed by direct counting and colocalization of nuclei and vessels. $J$ Neurosci 29,14553 14570, (2009).

74 Ke, M. T., Fujimoto, S. \& Imai, T. SeeDB: a simple and morphology-preserving optical clearing agent for neuronal circuit reconstruction. Nat Neurosci 16, 1154-1161, (2013).

75 Kermany, D. S. et al. Identifying Medical Diagnoses and Treatable Diseases by ImageBased Deep Learning. Cell 172, 1122-1131 e1129, (2018).

6 Biswas, S. \& Barma, S. A large-scale optical microscopy image dataset of potato tuber for deep learning based plant cell assessment. Sci Data 7, 371, (2020).

77 Yu, C. W., J.; Peng, C.; Gao, C.; Yu, G.; \& Sang, N. Bisenet: Bilateral segmentation network for real-time semantic segmentation. Proceedings of the European conference on computer vision (ECCV), 325-341, (2018).

78 Deng, J. D., W.; Socher, R.; Li, L.-J.; Li, K.; \& Fei-Fei, L. Imagenet: A large-scale hierarchical image database. IEEE conference on computer vision and pattern recognition, 248-255, (2009).

79 Bouet, V. et al. The adhesive removal test: a sensitive method to assess sensorimotor deficits in mice. Nat Protoc 4, 1560-1564, (2009).

80 Farr, T. D., Liu, L., Colwell, K. L., Whishaw, I. Q. \& Metz, G. A. Bilateral alteration in stepping pattern after unilateral motor cortex injury: a new test strategy for analysis of skilled limb movements in neurological mouse models. J Neurosci Methods 153, 104-113, (2006).

81 Metz, G. A. \& Whishaw, I. Q. The ladder rung walking task: a scoring system and its practical application. J Vis Exp, (2009).

82 Schallert, T., Fleming, S. M., Leasure, J. L., Tillerson, J. L. \& Bland, S. T. CNS plasticity and assessment of forelimb sensorimotor outcome in unilateral rat models of stroke, cortical ablation, parkinsonism and spinal cord injury. Neuropharmacology 39, 777-787, (2000).

83 Balkaya, M., Krober, J., Gertz, K., Peruzzaro, S. \& Endres, M. Characterization of longterm functional outcome in a murine model of mild brain ischemia. $J$ Neurosci Methods 213, 179-187, (2013). 
Acknowledgments:

806 Supported by operating grants from Canadian Institutes of Health Research (MOP-102501) and Heart and Stroke Foundation (G-16-00014016) awarded to Richard BERGERON.

We thank Dr. B Lacoste and his research associate Dr. X Toussay for their expertise in supervising and allowing us to use their Laser Doppler flowmeter. We also thank the following colleagues for discussions of the project in the last few years: P Albert, JC Béique, D Corbett, JT Coyle, RW Greene, A Hakim, M Khacho, B Lacoste, D Lagace, DG Rainnie, G Silasi, and G Tsai.

\section{Author Contributions:}

814 Performed and analyzed electrophysiological experiments: P.K., B.W., W.B., A.Y.C.W., S.R. 815 Purified and determined activity of glycine oxidase: N.A. Carried out behavioural testing and 816 surgical procedures: J.C., A.Y.C.W., A.S., P.K., B.W. Performed immunohistochemistry 817 experiments: J.C., A.S., A.Y.C.W. Generated viral constructs: P.C. Prepared cleared brain 818 samples: J.C. Constructed and operated the light sheet microscope: B.W., and J.P. Analyzed data imaged with the light sheet microscope: B.W., J.P., J.W. Performed and analyzed laser doppler flowmetry recordings: J.C., and A.Y.C.W. Provided and facilitated access to essential infrastructure: R.B. Supervised the study, performed experimental design and provided scientific direction: R.B. All authors provided advice on data interpretation and critical edits to the text and approved the final manuscript.

\section{We have no competing interest to declare}

\section{Additional information:}

Supplementary information is available for this paper

Correspondence and requests for materials should be addressed to:

Richard BERGERON

Ottawa Hospital Research Institute, 451 Smyth Road, Roger Guindon Building, Room 3210D,

835 Phone: 613-562-5800 \#3296;

836 Email: rbergeron@ohri.ca

837

838 Reprints and permissions information is available at www.nature.com/reprints 


\section{Figure 1}
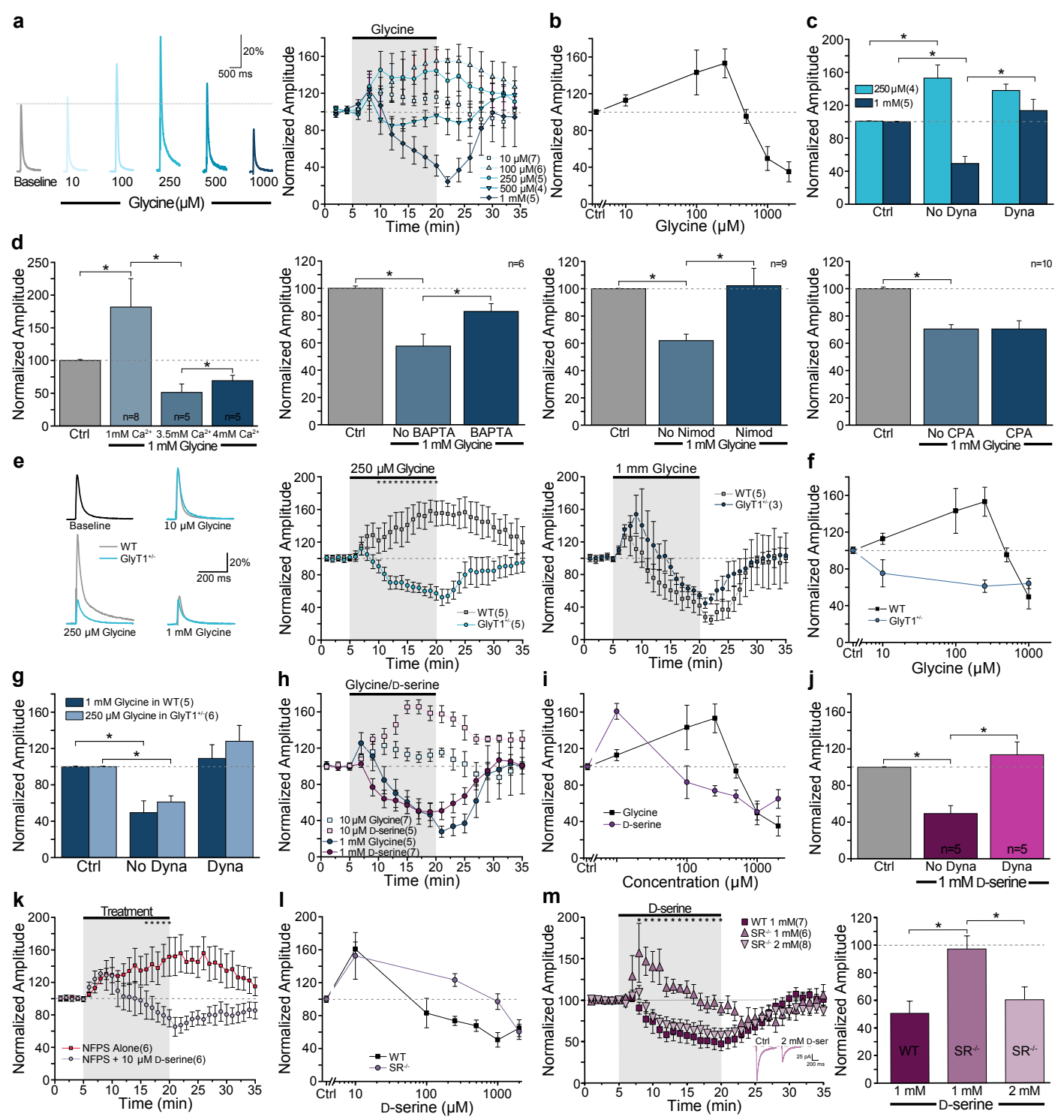

841 Figure 1: Increasing exogenous glycine and D-serine concentrations results in NMDAR

\section{internalization, which is dependent on $\mathrm{Ca}^{2+}$ influx.}

843 a) Normalized raw traces showing the effect of increasing concentrations of exogenous glycine on 844 Schaffer Collateral NMDAR-EPSCs and mean time-course data showing the effect of a $15 \mathrm{mins}$ application of various glycine concentrations. b) A dose-response curve of glycine and NMDAREPSC amplitudes. c) The effect of $250 \mu \mathrm{M}$ and $1 \mathrm{mM}$ glycine on NMDAR-EPSC amplitudes in the presence of dynasore. d) The role of various concentrations of extracellular $\mathrm{Ca}^{2+}$ on NMDAREPSC amplitudes in the presence of $1 \mathrm{mM}$ glycine, as well as changing intracellular $\mathrm{Ca}^{2+}$ levels using BAPTA, nimodipine, or CPA. e) The effect of various concentrations of glycine on NMDAR-EPSC amplitudes in GlyT1 ${ }^{+/-}$mice, compared to WT. f) The dose-response curve of the 
851 effects of glycine on NMDAR amplitudes in $\mathrm{GlyT}^{+/-}$mice. $\mathbf{g}$ ) The effect of $250 \mu \mathrm{M}$ glycine on 852 NMDAR-EPSC amplitudes in $\mathrm{GlyT}^{+/-}$mice in the presence of dynasore. h) The effect of low $853(10 \mu \mathrm{M})$ and high $(1 \mathrm{mM})$ concentrations of glycine or D-serine concentrations on NMDAR-EPSC 854 amplitudes. i) The dose-response curve of NMDAR-EPSC amplitudes to D-serine compared to 855 glycine. j) The effect of $1 \mathrm{mM}$ D-serine in the presence of dynasore. $\mathbf{k}$ ) The effect of $10 \mu \mathrm{M}$ D-serine 856 while elevating endogenous glycine levels with NFPS. I) A dose-response curve showing the effect 857 of exogenous D-serine levels on $\mathrm{SR}^{-/-}$mice. $\mathbf{m}$ ) The effect of a higher dose of D-serine ( $1 \mathrm{mM}$ vs. $8582 \mathrm{mM}$ ) on NMDAR-EPSC amplitudes in $\mathrm{SR}^{-/-}$mice compared to their WT littermates. Data is mean $859 \pm$ SEM; statistical significance $p<0.05 *$. 


\section{Figure 2}

a

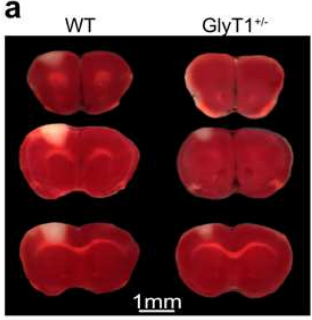

b

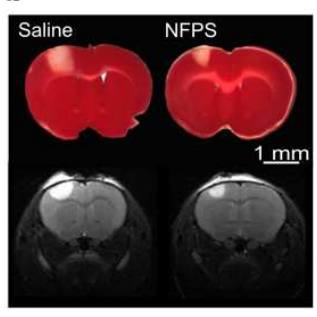

d
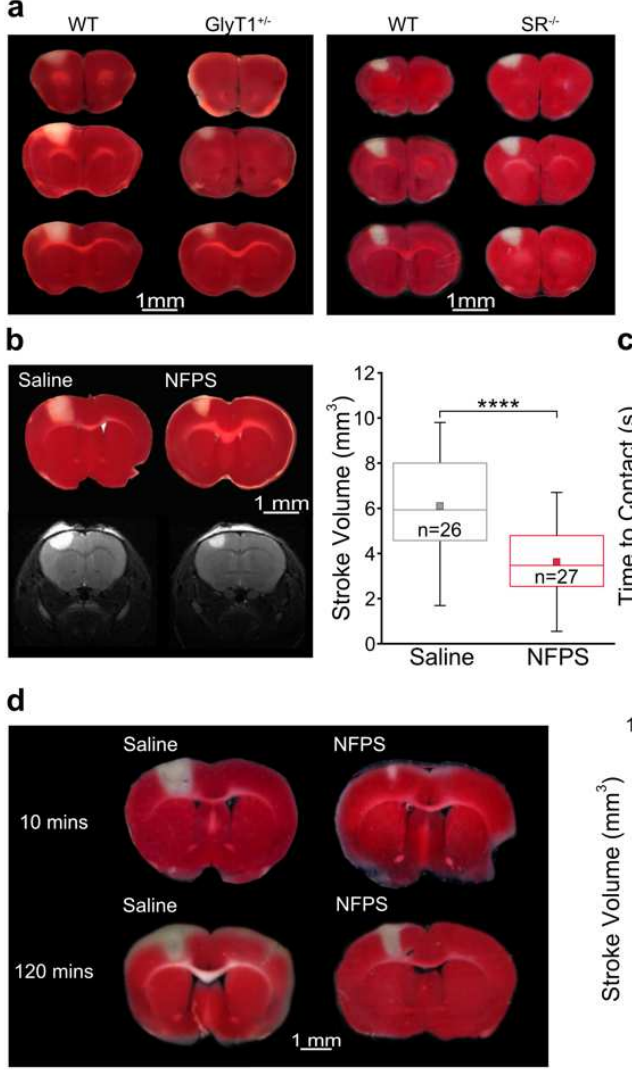
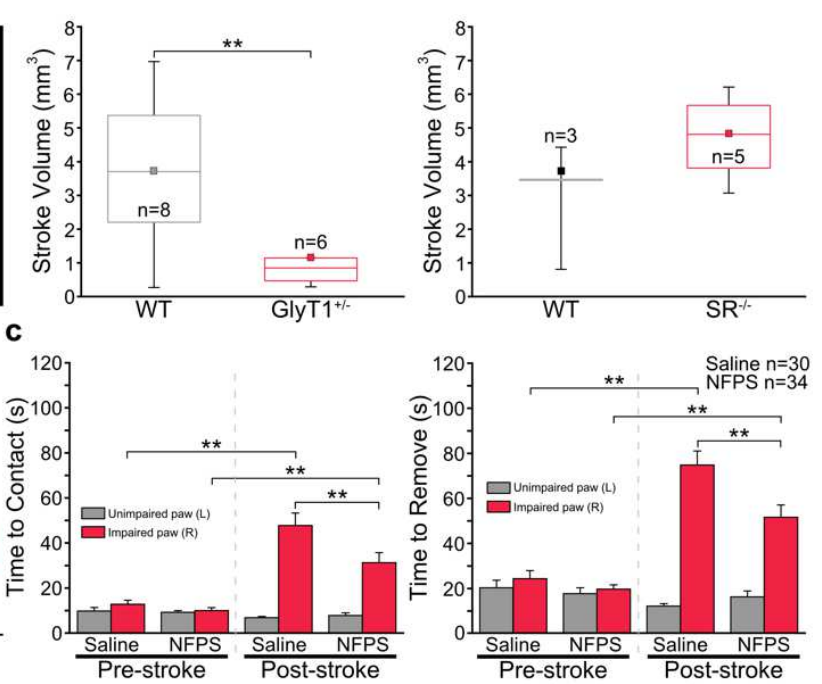

861

862

863

864

865

866

867

868

869

870

871
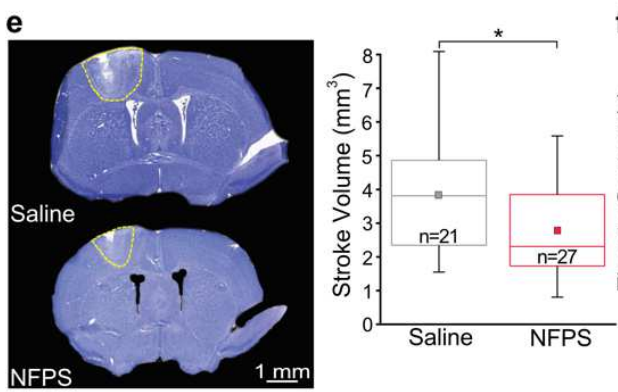

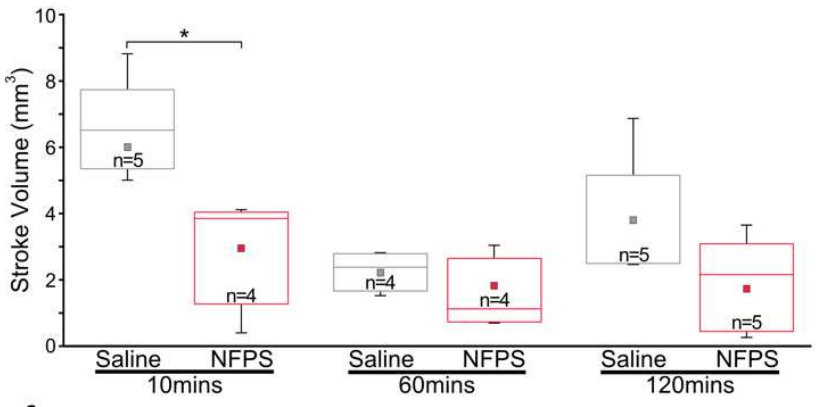

f

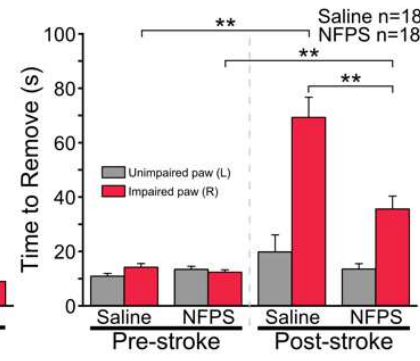

Figure 2: Elevation of extracellular glycine results in a smaller infarct volume and decreased motor behavioural deficits following photothrombotic and endothelin-1 stroke.

a) Representative serial coronal sections of TTC-stained mouse forebrain (slice thickness $500 \mu \mathrm{M}$ ) and their corresponding box and whisker plots showing the infarct volume when assessed $48 \mathrm{hrs}$ after the induction of a unilateral photothrombotic stroke in $\mathrm{GlyT}^{+/-}$and $\mathrm{SR}^{-/-}$mice relative to WT mice. b) Representative TTC-stained (top) or magnetic resonance imaging (bottom) sections showing representative stroke regions observed $48 \mathrm{hrs}$ following the induction of photothrombotic (PT) stroke, and a box and whisker plot showing stroke volume in saline-treated or NFPS-treated mice $24 \mathrm{hrs}$ prior to stroke induction. c) The effect of 24hrs pre-stroke NFPS administration on 
872 post-stroke time to contact and time to remove in the adhesive removal task compared with saline 873 treatment, when evaluated $48 \mathrm{hrs}$ following PT stroke. d) Effect of various post-stroke 874 administration time-points of NFPS treatment on stroke volume with their corresponding box and

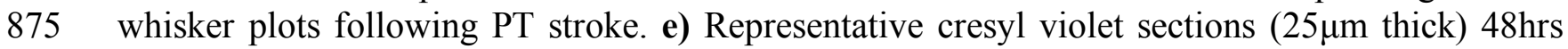
876 following endothelin-1 (ET-1) stroke obtained from saline-treated and NFPS-treated mice 24hrs 877 prior, in which the extent of the infarct is shown within the yellow border and box and whisker 878 plot depicting infarct volume. f) The effect of $24 \mathrm{hrs}$ pre-stroke NFPS administration on post-stroke 879 time to contact and time to remove in the adhesive removal task compared with saline treatment 880 following ET-1 stroke. Data is mean $\pm \mathrm{SEM}$; statistical significance $p<0.05 *, p<0.01 * *, p<$ $8810.001 * * *$, and $p<0.0001 * * * *$. 


\section{Figure 3}
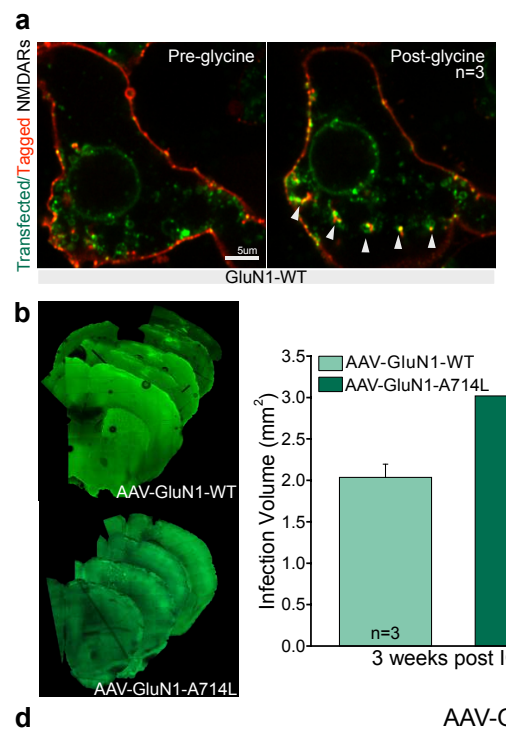

d

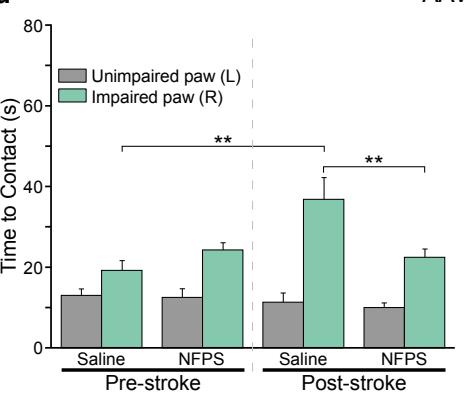

e

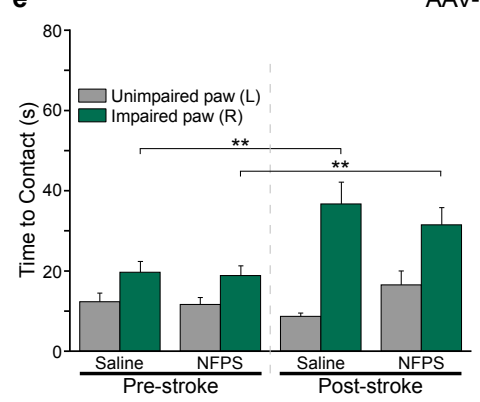

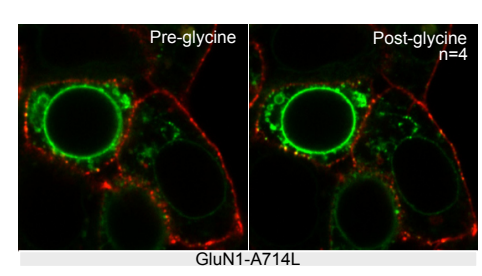

C
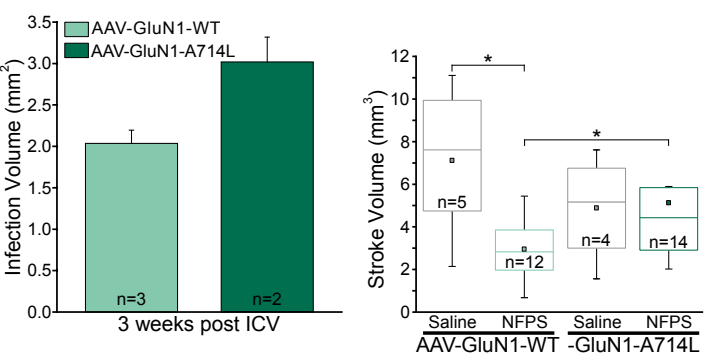

AAV-GluN1-WT
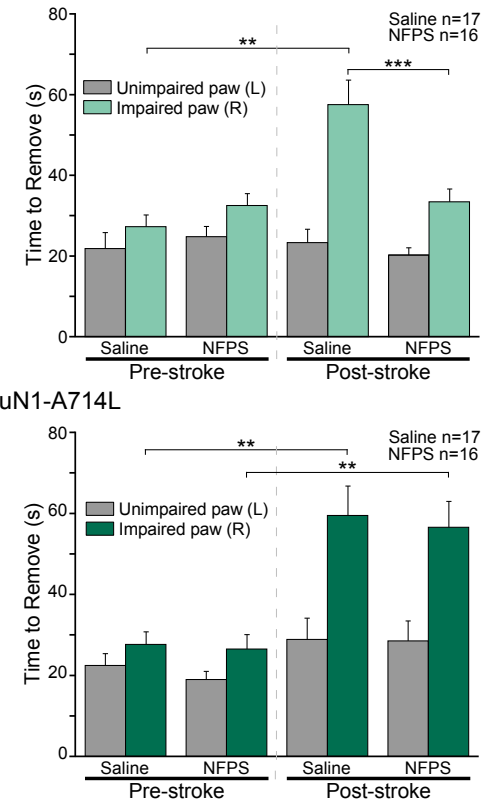

Figure 3: Infection of the stroke site with the non-internalizing GluN1-A714L mutation abolishes the protective effect of elevating extracellular glycine on stroke volume and during a behavioural task.

a) Visual representation of NMDAR internalization in GluN1-WT or GluN1-A714L transfected HEK293 cells following application of $1 \mathrm{mM}$ glycine. Transfected NMDARs are labeled in green, while extracellular NMDARs are additionally labeled with red cell impermeable nanobody staining. b) Representative images showing the extent of viral spread in the mouse forebrain following infection between mice infected with AAV-GluN1-WT or AAV-GluN1-A714L. c) Box and whisker plot showing the effect of NFPS administration 24hrs prior to PT stroke induction in mice infected with AAV-GluN1-WT or AAV-GluN1-A714L. d-e) The effect of NFPS on post- 
894 stroke time to contact and time to remove in the adhesive removal task compared with saline 895 treatment, in mice infected with AAV-GluN1-WT (d), and in mice infected with AAV-GluN1-

896 A714L (e) $48 \mathrm{hrs}$ following PT stroke. Data is mean \pm SEM; statistical significance $p<0.05^{*}, p<$ $8970.01 * *$, and $p<0.001 * * *$. 
Figure 4

a

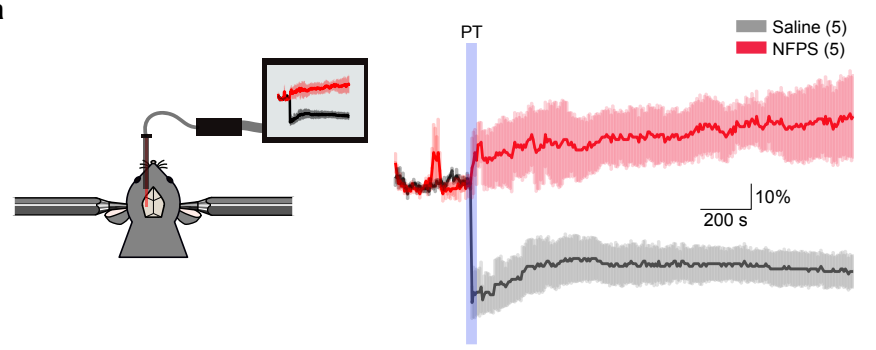

c
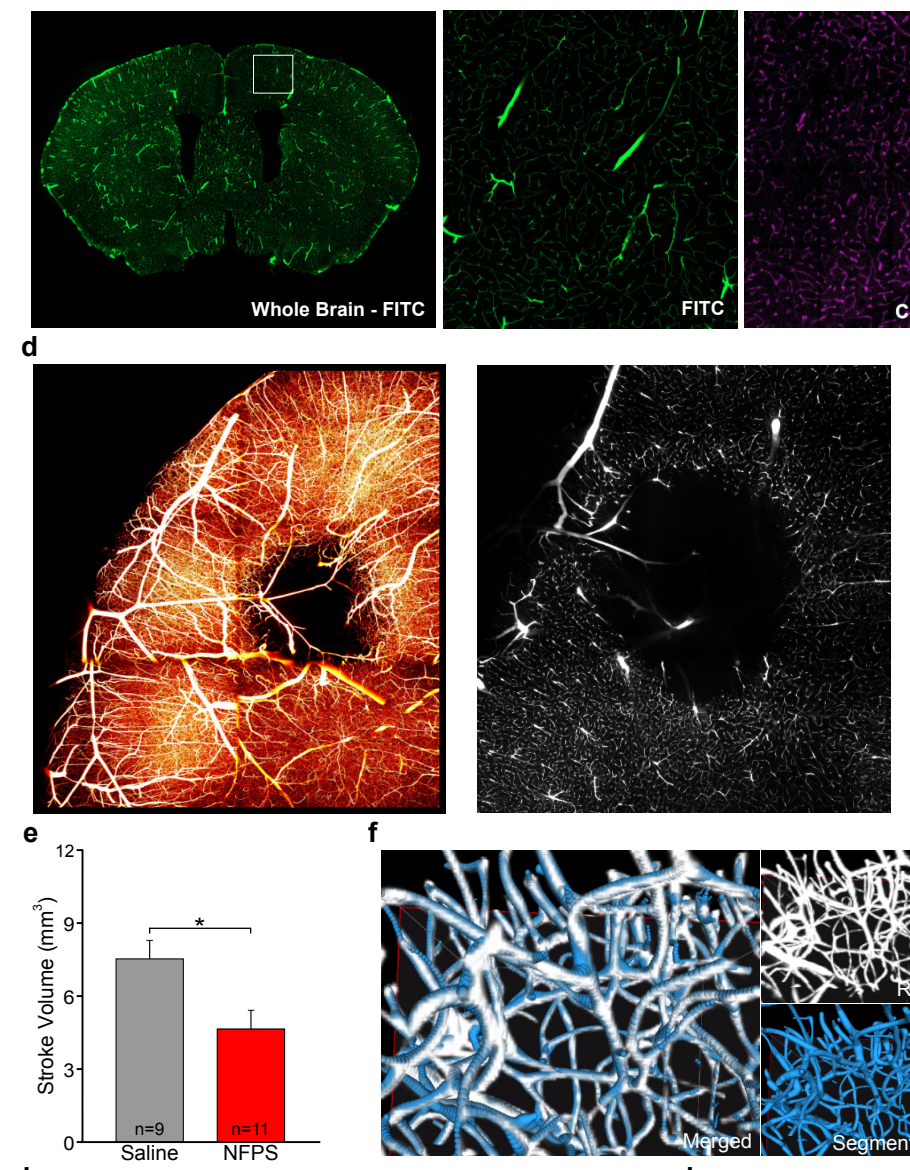

h
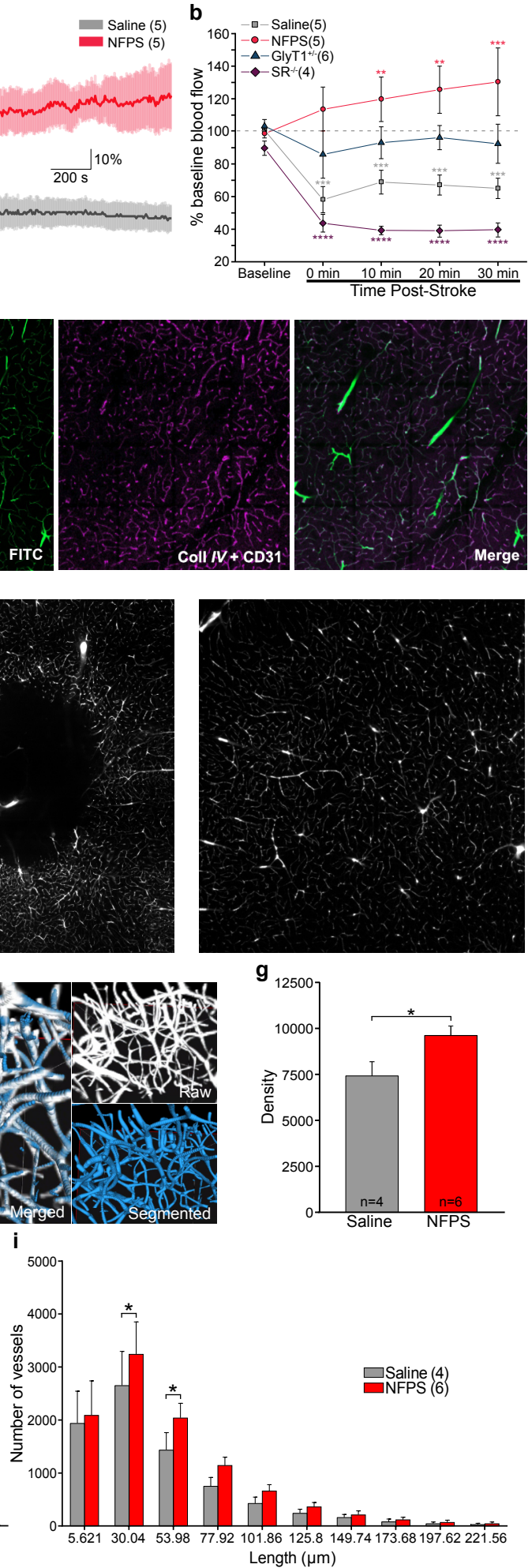
901 Figure 4: Laser doppler flowmetry blood flow and automatic segmentation of vascular data 902 with AIVIA 9.

903 a) Diagram depicting laser doppler flowmetry set-up and the measured effect of NFPS on cerebral 904 blood flow following photothrombotic (PT) stroke. b) Time-course of post-stroke cerebral blood 905 flow following PT in mice with varying levels of glycine or D-serine. c) $50 \mu \mathrm{m}$ coronal section of 906 brain perfused with FITC-BSA. Magnified images from the sensorimotor cortex demonstrating 907 exact colocalization of FITC-BSA perfusion (green) with CD31 and Coll $I V$ vascular 908 immunostaining (purple). d) Colorized max projection of stroked hemisphere, and single section 909 of raw images depicting vasculature at the stroke and below the stroke, acquired with a light sheet 910 microscope 48hrs following PT stroke. e) Stroke volume bar graph in saline- or NFPS-treated 911 mice, calculated by an automated deep learning prediction model. f) Merged image demonstrating 912 exact colocalization of AIVIA's automatic segmentation to raw data. g) Density of vessels in peri913 infarct region in saline- or NFPS-treated mice. h-i) Number of vessels in the stroke area according 914 to diameter and to length. Data is mean \pm SEM; statistical significance $p<0.05^{*}, p<0.01 * *, p$ $915<0.001 * * *$, and $p<0.0001 * * * *$. 


\section{Extended Data Figure 1}
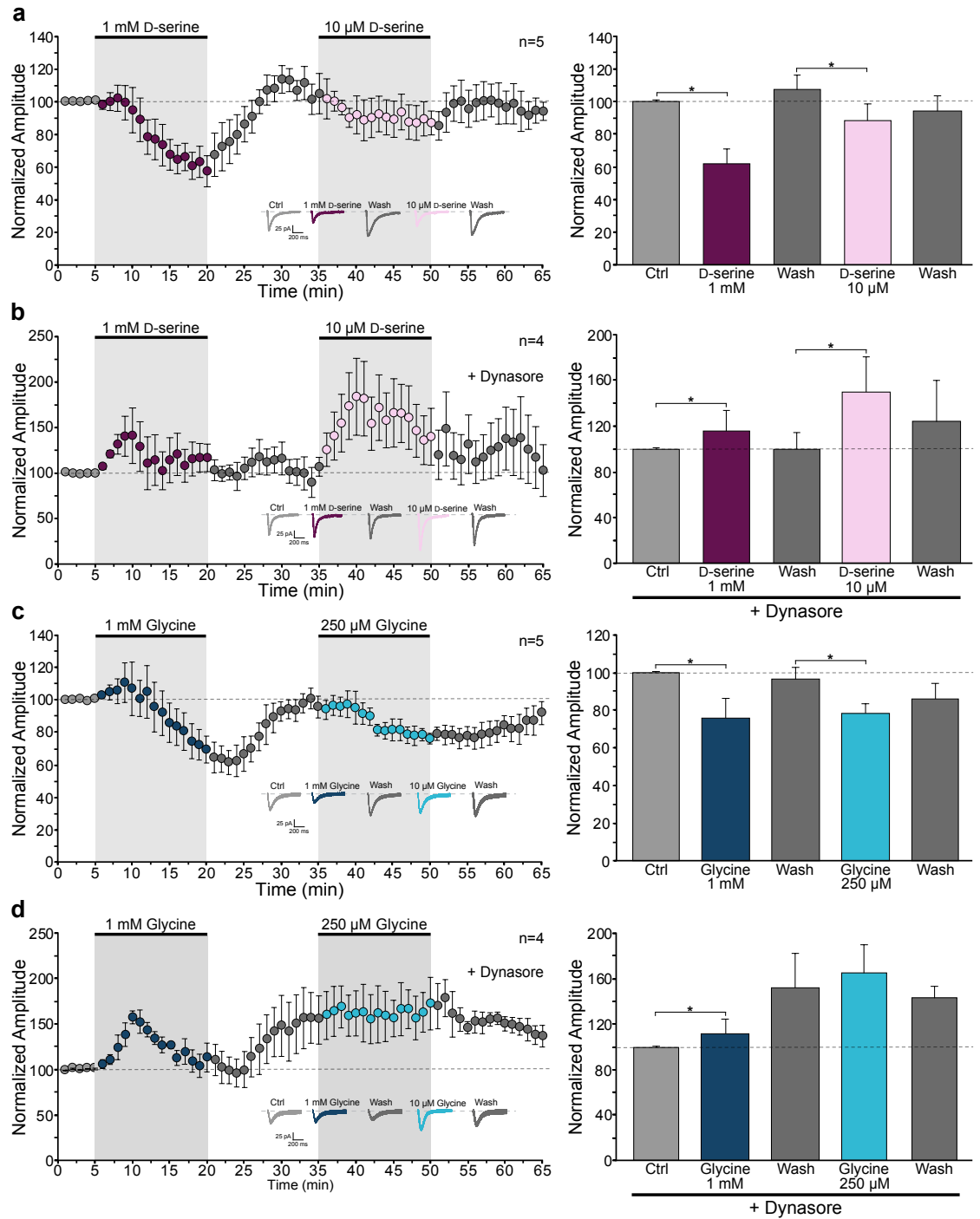

Extended Data Figure 1: Priming NMDARs with a high dose of glycine or D-serine allows glycine-induced NMDAR internalization to occur at a low dose of glycine or D-serine. a) Mean time-course data showing the continuous application of high dose $(1 \mathrm{mM})$ D-serine followed by low dose $(10 \mu \mathrm{M})$ D-serine in WT mice on NMDAR-EPSCs and its corresponding bar graph. b) Same as a) in the presence of dynasore. c) Mean time-course data showing the continuous application of high dose $(1 \mathrm{mM})$ glycine followed by low dose $(250 \mu \mathrm{M})$ glycine in WT mice on NMDAR-EPSCs and its corresponding bar graph. d) Same as c) in the presence of dynasore. Data is mean $\pm \mathrm{SEM}$; statistical significance $p<0.05 *$. 


\section{Extended Data Figure 2}
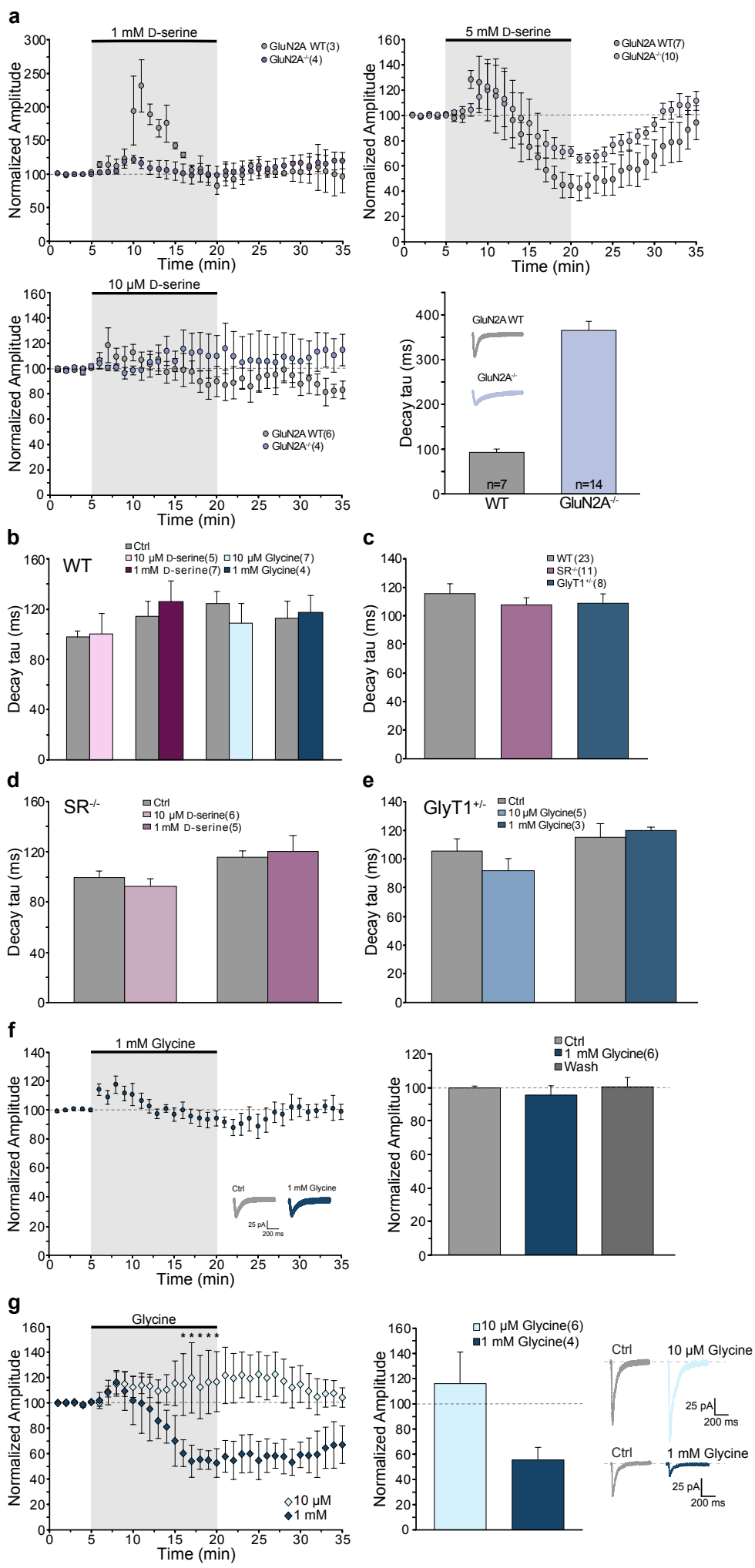
927 Extended Data Figure 2: Glycine-induced NMDAR internalization is not subunit-specific, 928 nor dependent on AMPA receptor activation, and this effect is not restricted to the 929 hippocampus.

930 a) Mean time-course data showing the effect of $15 \mathrm{mins}$ application of $1 \mathrm{mM}, 5 \mathrm{mM}$ and $10 \mu \mathrm{M}$ of 931 exogenous D-serine on NMDAR-EPSCs in WT and GluN2 $\mathrm{A}^{-/-}$mice. Note: The mouse strain with 932 a different background $(\mathrm{C} 57 \mathrm{Bl} / 6)$ required a higher dose of D-serine to initiate NMDAR 933 internalization, hence the $5 \mathrm{mM}$ D-serine application. NMDAR decay kinetics in WT and GluN2A-

934 /- mice was also measured to verify the lack of GluN2A subunit-containing NMDARs and this was 935 made evident with the slower decay tau. b-e) NMDAR decay kinetics in WT, $\mathrm{SR}^{-/}$, and GlyT1 ${ }^{+/-}$ 936 mice following application of $1 \mathrm{mM}$ or $10 \mu \mathrm{M}$ glycine or D-serine. No differences in decay tau were 937 seen in any of the conditions. f) Mean time-course data showing the effect of 15 mins application 938 of $1 \mathrm{mM}$ of exogenous glycine on AMPA receptor-EPSCs and corresponding bar graph; 939 internalization was not observed. g) Mean time-course data showing the effect of $15 \mathrm{mins}$ 940 application of $1 \mathrm{mM}$ and $10 \mu \mathrm{M}$ of exogenous glycine on NMDAR-EPSCs recorded from the cortex 941 with its corresponding bar graph and normalized raw data traces. Data is mean \pm SEM; statistical 942 significance $p<0.05 *$. 


\section{Extended Data Figure 3}

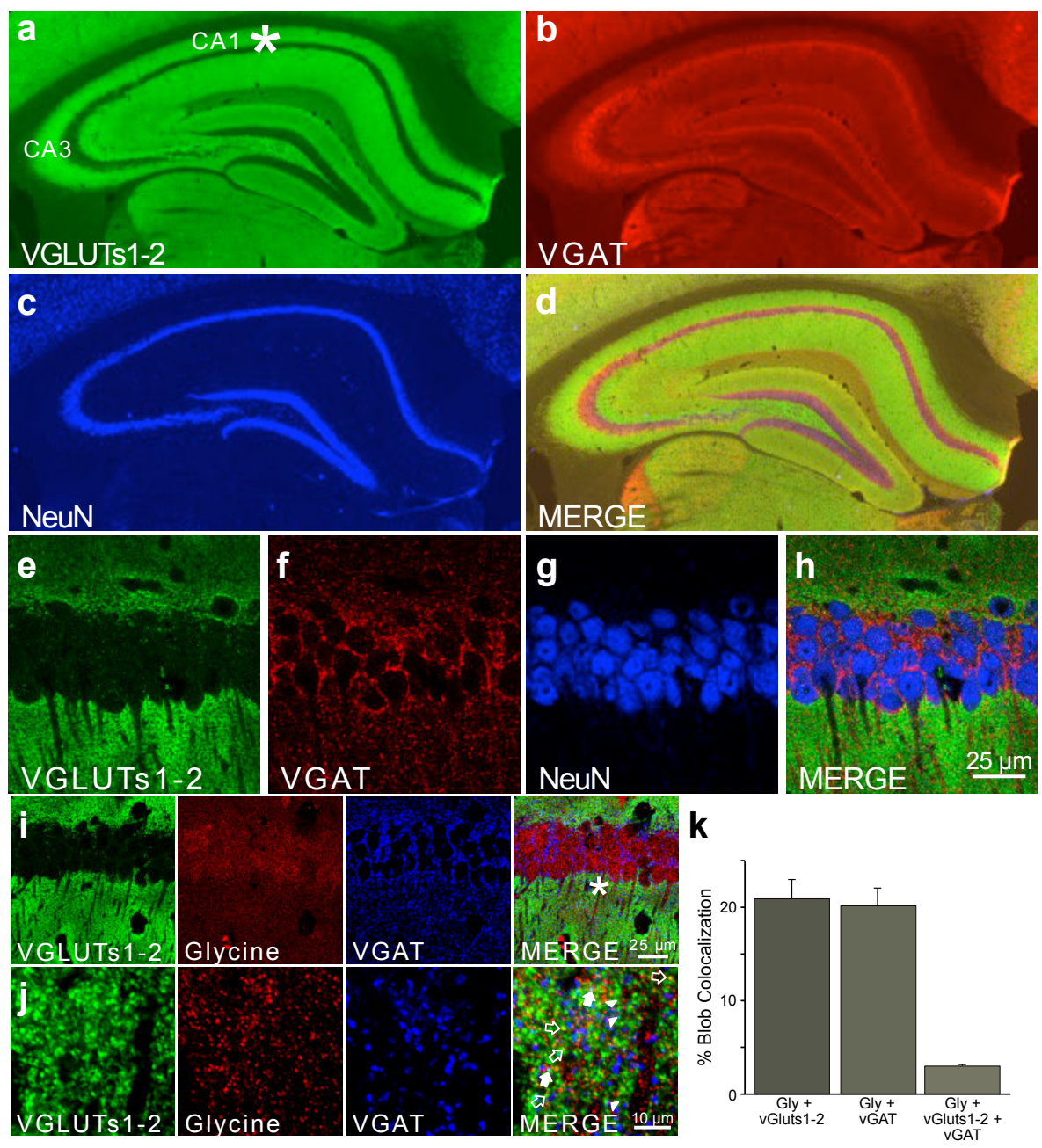

Extended Data Figure 3: Glycine is co-localized with markers for glutamatergic terminals in the CA1 region of the hippocampus.

947 a-d) Confocal images of a hippocampal slice with triple immunohistological co-staining showing the localization of vesicular glutamate transporters 1 and 2 (a; VGLUTs1-2; green), vesicular inhibitory amino acid transporters (b; VGAT; red) and the neuronal cell body marker NeuN (c; blue), and the three-channel merged composite image (d). e-h) High magnification images of the asterisked region in panel a, showing segregation of VGLUTs1-2 to extrasomatic regions (e), and VGATs to somatic regions (f), separated by neuronal cell bodies (g; NeuN). Merged image shown in h. i) High magnification images of the CA1 region of a different slice co-stained with antibodies against VGLUTs 1-2 (green), glycine (red) and VGAT (blue). j) Zoomed image of the perisomatic region illustrated by the asterisk shown in $\mathbf{g}$, illustrating the region of interest used for triple blob 


\section{Extended Data Figure 4}
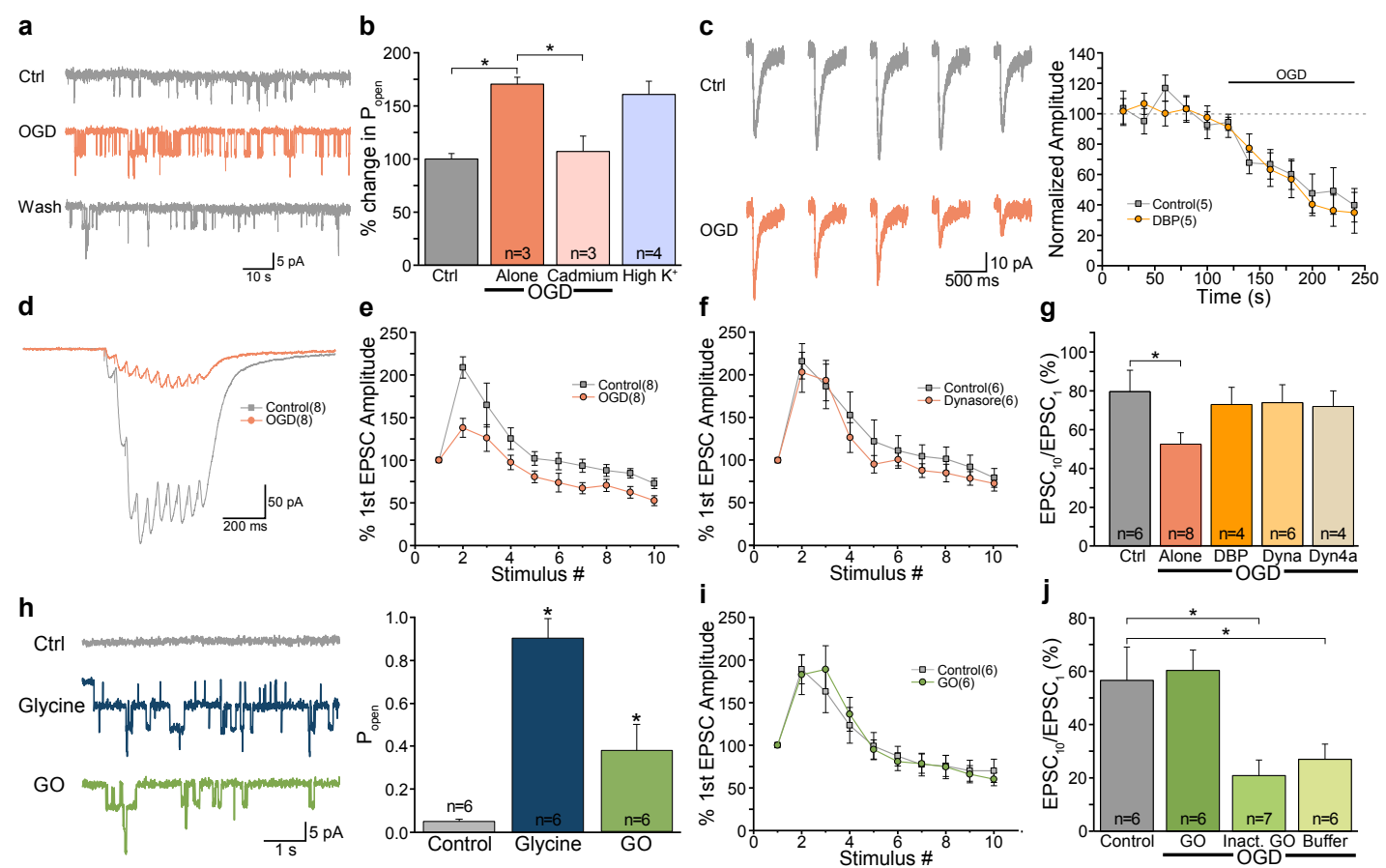

Extended Data Figure 4: Glycine-induced NMDAR internalization is triggered during oxygen-glucose deprivation conditions and is blocked by dynamin and glycine oxidase.

a) The effect of oxygen-glucose deprivation (OGD) on $P_{\text {open }}$ of homomeric $\langle 2$ glycine receptors. b) Changes in $P_{\text {open }}$ in the presence of OGD alone or $300 \mu \mathrm{M}$ cadmium, a non-specific $\mathrm{Ca}^{2+}$ channel blocker. The robust response to OGD was abolished suggesting a $\mathrm{Ca}^{2+}$-dependent glycine release process. As a positive control, $35 \mathrm{mM} \mathrm{K}^{+}$was applied to the slice to induce glycine release via neuronal depolarization and a comparable increase in $P_{\text {open }}$ was observed. c) Raw traces showing the effect of OGD on NMDAR-EPSCs evoked at low-frequency and the normalized NMDAREPSC amplitudes during OGD in the presence of dynamin blocking peptide (DBP), which inhibits NMDAR internalization. The OGD paradigm induced a decrease in the amplitude of NMDAREPSCs during the single pulse stimulation paradigm. Application of DBP did not attenuate the effect of OGD. Therefore, the decreased NMDAR-EPSC amplitudes resulting from single pulse stimulation of the presynaptic terminal was not due to GINI d-e) The effect of OGD on NMDAREPSC amplitudes evoked by a $20 \mathrm{~Hz}, 10$ stimulus train, compared with control. When the amplitude of the last EPSC in the train $\left(\mathrm{EPSC}_{10}\right)$ was normalized to the first amplitude of the train $\left(\mathrm{EPSC}_{1}\right)$, the $\mathrm{EPSC}_{10} / \mathrm{EPSC}_{1}$ ratio, there was a significant smaller ratio during OGD than in control conditions, likely due to NMDAR internalization. $\mathbf{f}-\mathbf{g}$ ) The effect of OGD on $\mathrm{EPSC}_{10} / \mathrm{EPSC}_{1}$ ratio for trains elicited in OGD conditions in the presence of various internalization blockers, DBP, dynasore (dyna), and Dyngo4a (Dyn4a), showing similar results to that observed in control conditions. h) The effect of purified glycine oxidase (GO; an enzyme that catalyzes the breakdown of glycine) with $1 \mu \mathrm{M}$ glycine on $P_{\text {open }}$ of homomeric $\langle 2$ glycine receptors expressed in outside-out patches obtained from $\mathrm{CHO}$ cells during OGD. $\mathbf{i}-\mathbf{j}$ ) The presence of GO abolished the combined 
983 inhibitory effect of OGD and a $20 \mathrm{~Hz}$ train on NMDAR-EPSC amplitudes. Data is mean $\pm \mathrm{SEM}$; 984 statistical significance $p<0.05 *$. 


\section{Extended Data Figure 5}
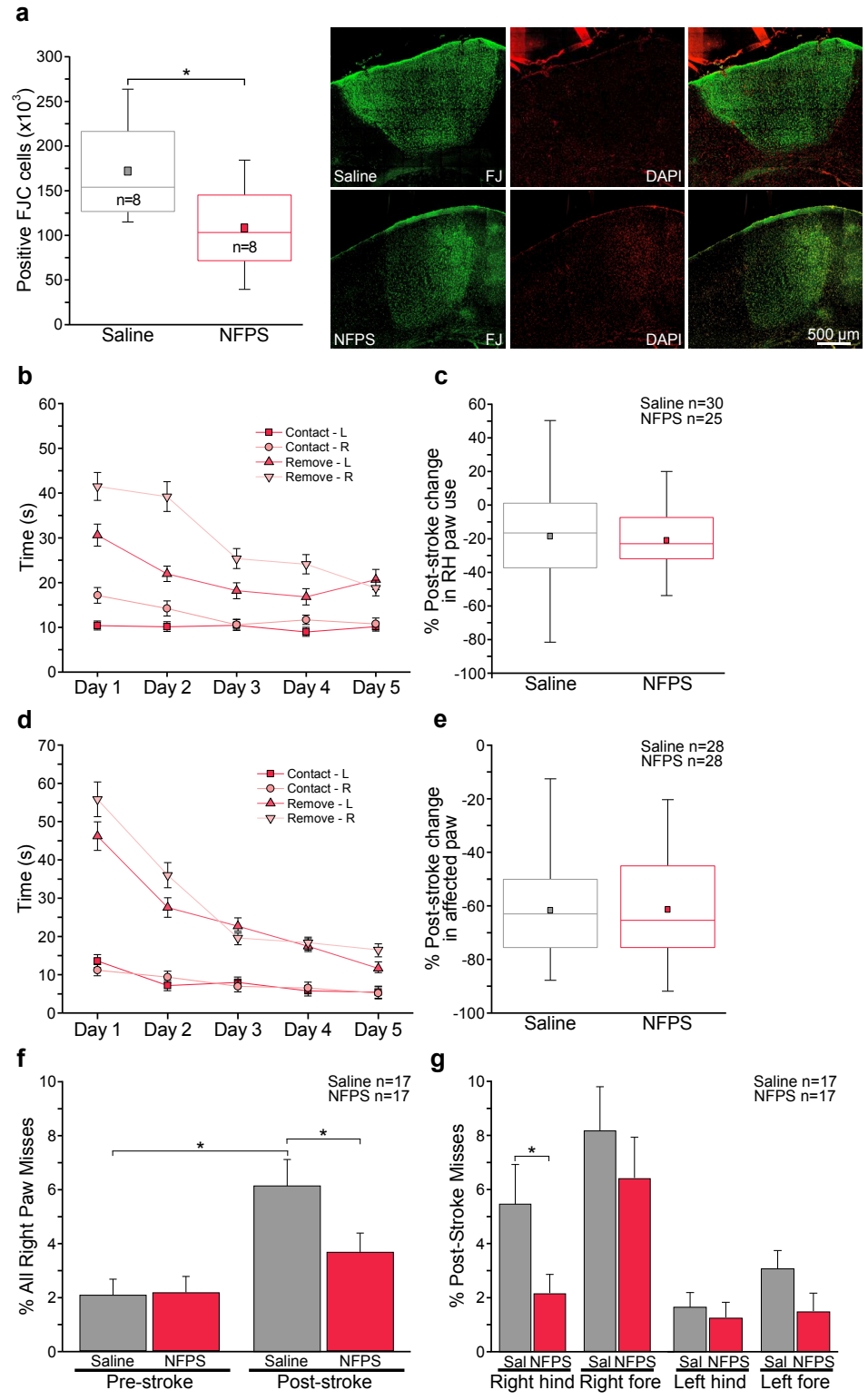

Extended Data Figure 5: Supplementary data of the effect of NFPS administration on cell death and behavioural tasks following photothrombosis and endothelin-1 strokes.

989 a) Cell death assay (Fluoro Jade C; green) on photothrombotic (PT) stroke following NFPS administration. b) Training curves for the adhesive task following PT stroke with NFPS. c) Cylinder task data following NFPS administration and PT stroke. d) Training curves for the adhesive task following endothelin-1 (ET-1) stroke with NFPS. e) Cylinder task data following NFPS administration and ET-1 stroke. $\mathbf{f}-\mathbf{g}$ ) The effect of pre-stroke NFPS administration on poststroke deficits in the horizontal ladder test following ET-1 stroke; percentage of misses both preand post- ET-1 stroke for mice treated with saline or NFPS is depicted. Data is mean \pm SEM; statistical significance $p<0.05^{*}, p<0.01^{* *}, p<0.001 * * *$, and $p<0.0001 * * * *$. 


\section{Extended Data Figure 6}

a
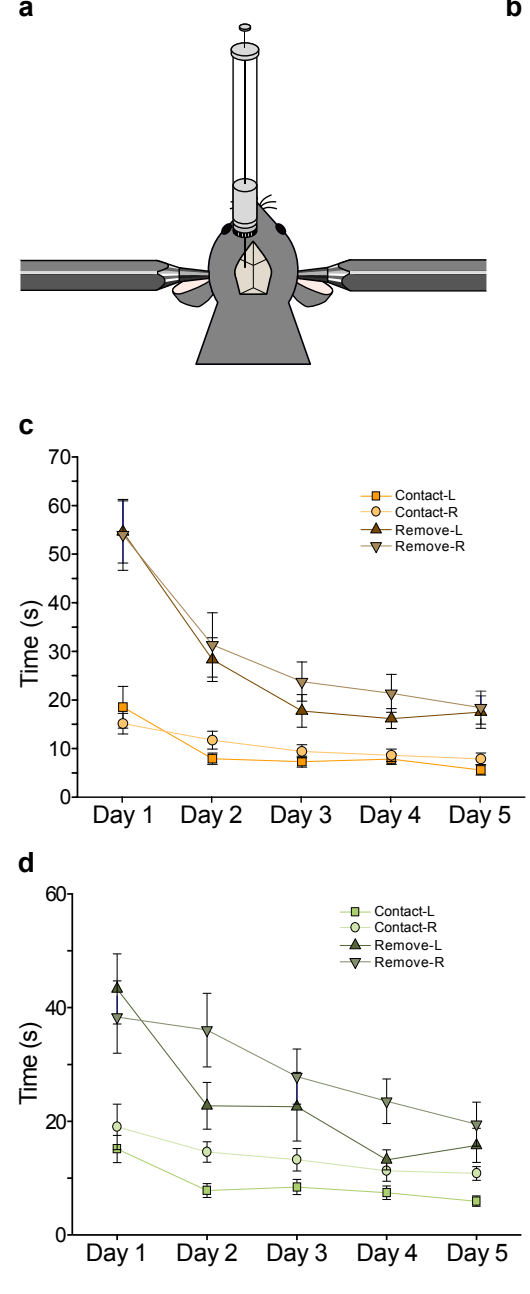

b
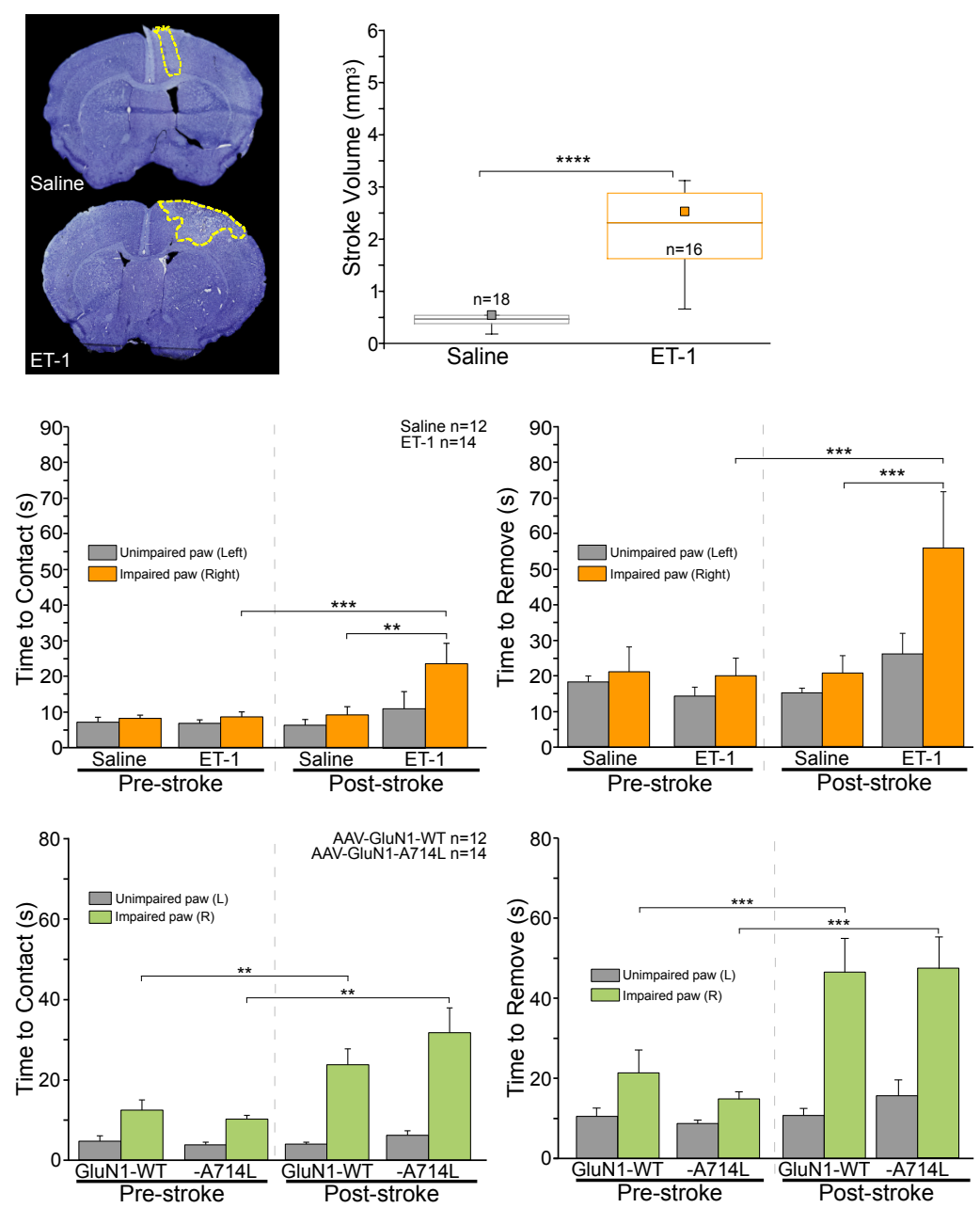

e

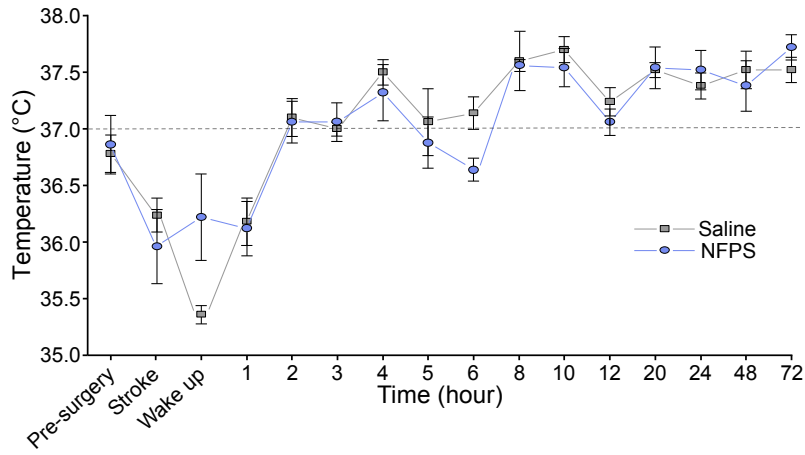

\section{Extended Data Figure 6: Control data for in vivo experiments.}

a-c) Endothelin-1 injection induced stroke and behavioural deficits in the adhesive removal task, compared to sham stroke (saline injection). d) Effect of adeno-associated virus (AAV)-GluN1WT and AAV-GluN1-A714L intracortical injection on post-stroke behavioural performance in the 
1003 adhesive removal task. e) Temperature time-course experiment following photothrombotic stroke, 1004 showing no contribution of hypothermia on the therapeutic effect of NFPS. Data is mean $\pm \mathrm{SEM}$; 1005 statistical significance $p<0.05^{*}, p<0.01^{* *}, p<0.001 * * *$, and $p<0.0001 * * * *$. 


\section{Extended Data Figure 7}
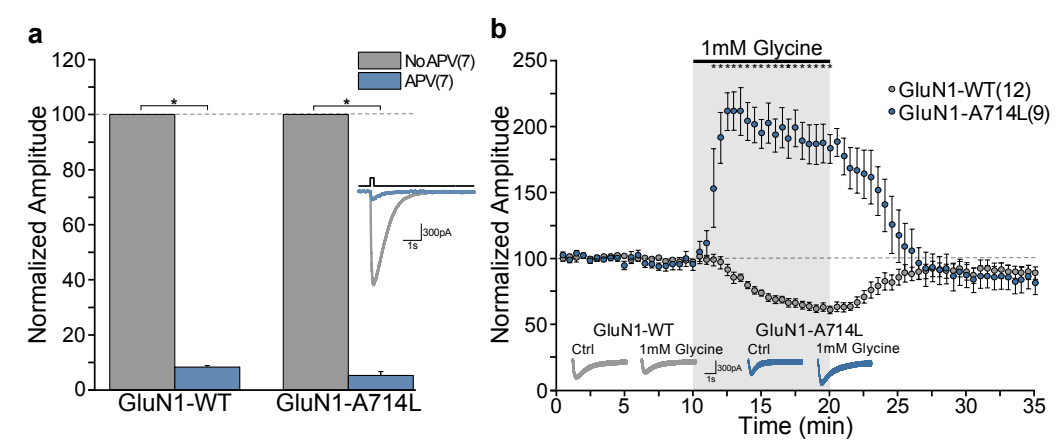

c
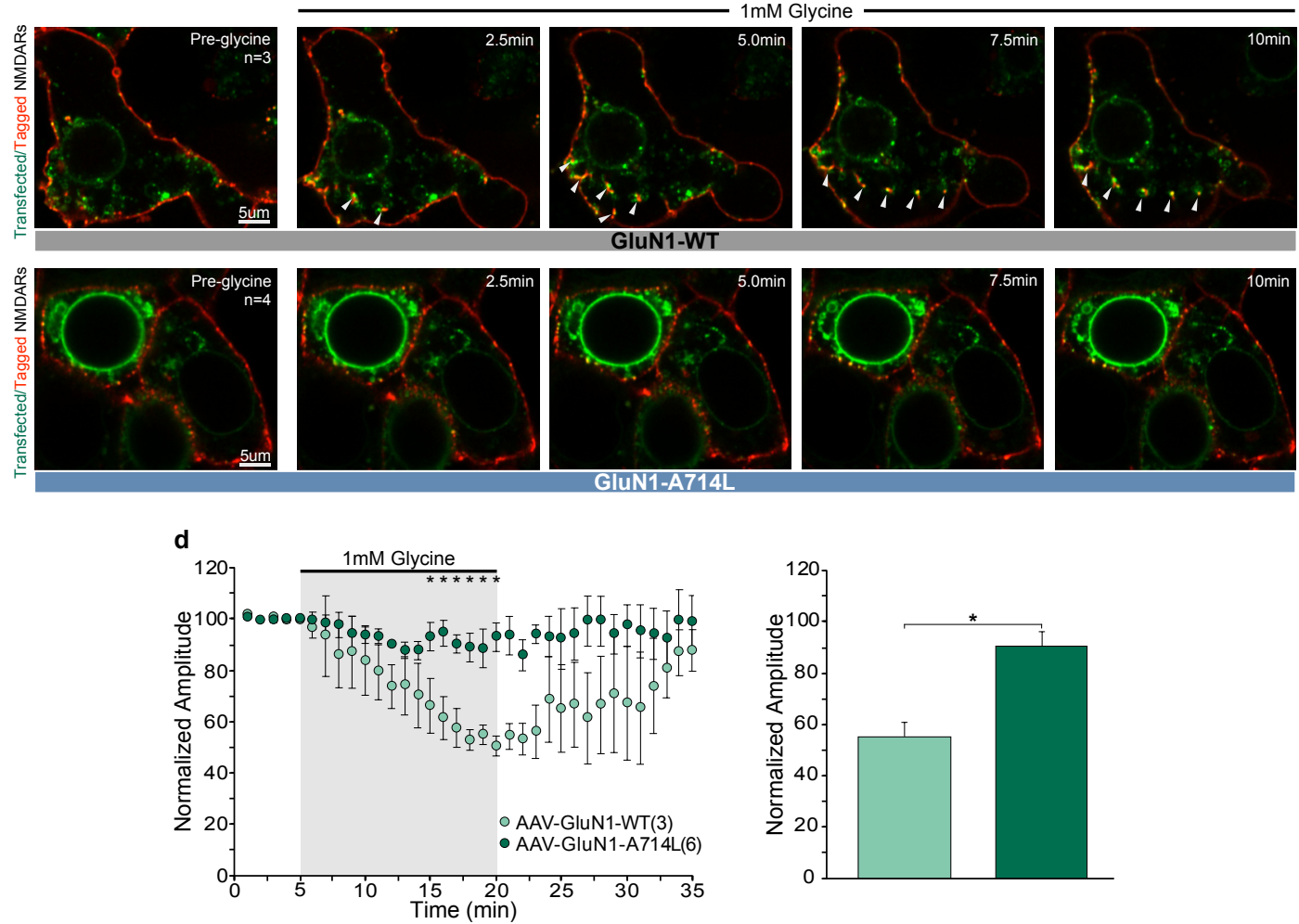

1006

1007

1008

1009

1010

1011

1012

1013

1014

1015

1016

1017

1018
Extended Data Figure 7: Electrophysiological representation and live-cell imaging visualization of glycine-induced NMDAR internalization blockade following application of $1 \mathrm{mM}$ glycine.

a) Normalized NMDAR-EPSC amplitudes and normalized raw data traces recorded from GluN1WT and GluN1-A714L transfected HEK293 cells with and without the NMDAR competitive antagonist, APV. b) Normalized NMDAR-EPSC amplitudes mean time-course data showing the effect of 1mM glycine application in GluN1-WT and GluN1-A714L transfected HEK293 cells. c) Visual representation of the internalization of NMDARs into the cell in GluN1-WT and GluN1A714L transfected HEK293 cells Following application of $1 \mathrm{mM}$ glycine, internalization of NMDARs was observed in the GluN1-WT transfected HEK293 cells but not in the GluN1-A714L transfected cells. Transfected NMDARs are labeled in green, while extracellular NMDARs are 
1019 additionally labeled in red, with cell impermeable nanobody. d) Normalized NMDAR-EPSC 1020 amplitudes mean time-course data and bar graph showing the effect of $1 \mathrm{mM}$ glycine application 1021 on cortical slices in mice infected with either the AAV-GluN1-WT or AAV-GluN1-A714L 1022 constructs. Data is mean \pm SEM; statistical significance $p<0.05 *$. 


\section{Extended Data Figure 8}
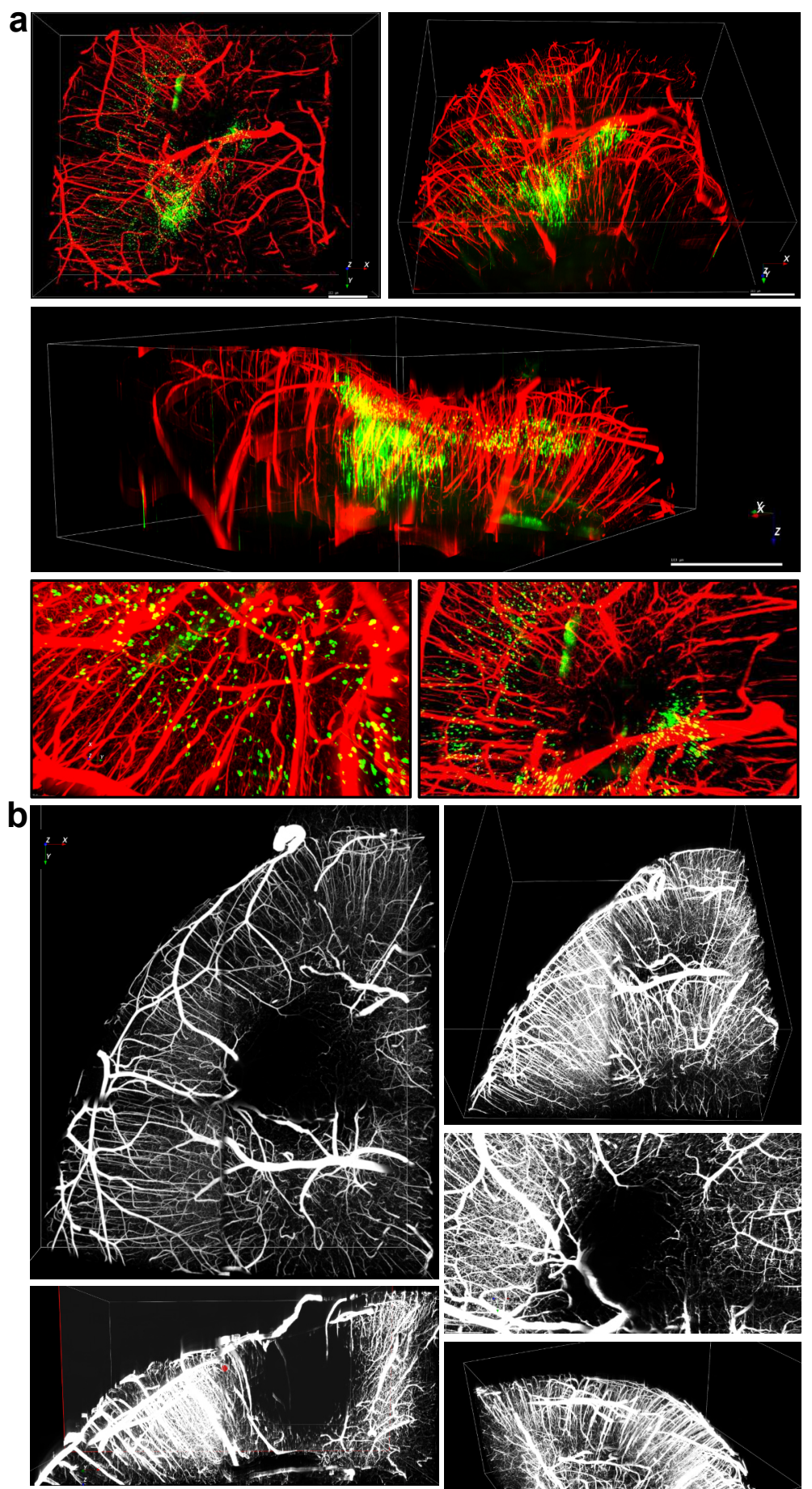

$\rightarrow-1,45,2$

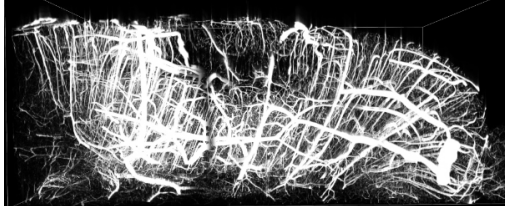

-

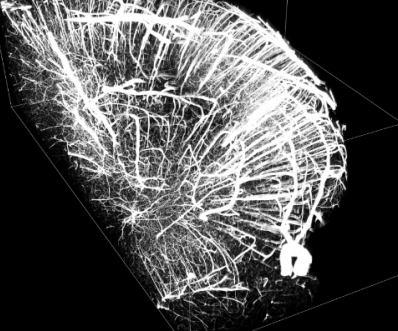


1024 Extended Data Figure 8: 3D vascular imaging and viral infection visualization.

1025 a) Various angles from 3D datasets acquired with the light sheet microscope demonstrating 1026 vasculature (red) and extent of viral infection (green) following injection of the adeno-associated 1027 virus GluN1-WT in the left hemisphere of a mouse having been induced by a photothrombotic 1028 stroke. b) Various angles from 3D datasets acquired with the light sheet microscope demonstrating 1029 vasculature in the left hemisphere of a mouse having been induced a photothrombotic stroke. 


\section{Extended Data Table 1}

\begin{tabular}{|l|c|c|}
\hline Primary Antibody & Source & Dillution \\
\hline Glycine (rabbit) & Abcam & $1: 100$ \\
\hline Synaptophysin (mouse) & Millipore Sigma & $1: 1000$ \\
\hline $\begin{array}{l}\text { Vesicular glutamate transporter 1 and 2 } \\
\text { (VGLUT1 and VGLUT2; guinea pig) }\end{array}$ & Millipore Sigma & $1: 1000$ \\
\hline $\begin{array}{l}\text { Vesicular GABA transporter } \\
\text { (VGAT; mouse) }\end{array}$ & Synaptic Systems & $1: 250$ \\
\hline CD31 (rabbit) & Abcam & $1: 50$ \\
\hline Collagen IV (rabbit) & Abcam & $1: 400$ \\
\hline Neuronal nuclei (mouse) & Millipore Sigma & $1: 1000$ \\
\hline Secondary Antibody & Source & Dillution \\
\hline CY3 (donkey anti-rabbit & Jackson ImmunoResearch & $1: 800$ \\
\hline Alexa-647 (donkey anti-mouse) & Molecular Probes & $1: 200$ \\
\hline FITC (goat anti-guinea pig) & Jackson ImmunoResearch & $1: 100$ \\
\hline
\end{tabular}

Extended Data Table 1. List of antibodies. 


\section{Extended Data Table 2}

\begin{tabular}{l|l|}
\hline Primer & Sequence \\
\hline NhelGluNI-F & 5'-AGCTAGCATGAGCACCATGCACCTGCT-3' \\
\hline EcoRVGluN1-R & 5'-AGATATCTCAGCTCTCCCTATGACGGG-3' \\
\hline A714L-F & 5'P-ACAATTACGAGAGCCTGGCTGAGGCCATCCA-3' \\
\hline A714L-R & 5'P-GCTCTCGTAATTGTGTTTTCCATGTGCCG-3' \\
\hline
\end{tabular}

1032

1033 Extended Data Table 2. Primers used for GluN1 variants subcloning into pcDNA3.1. F indicates 1034 a forward primer, $\mathrm{R}$ indicates a reverse primer. 

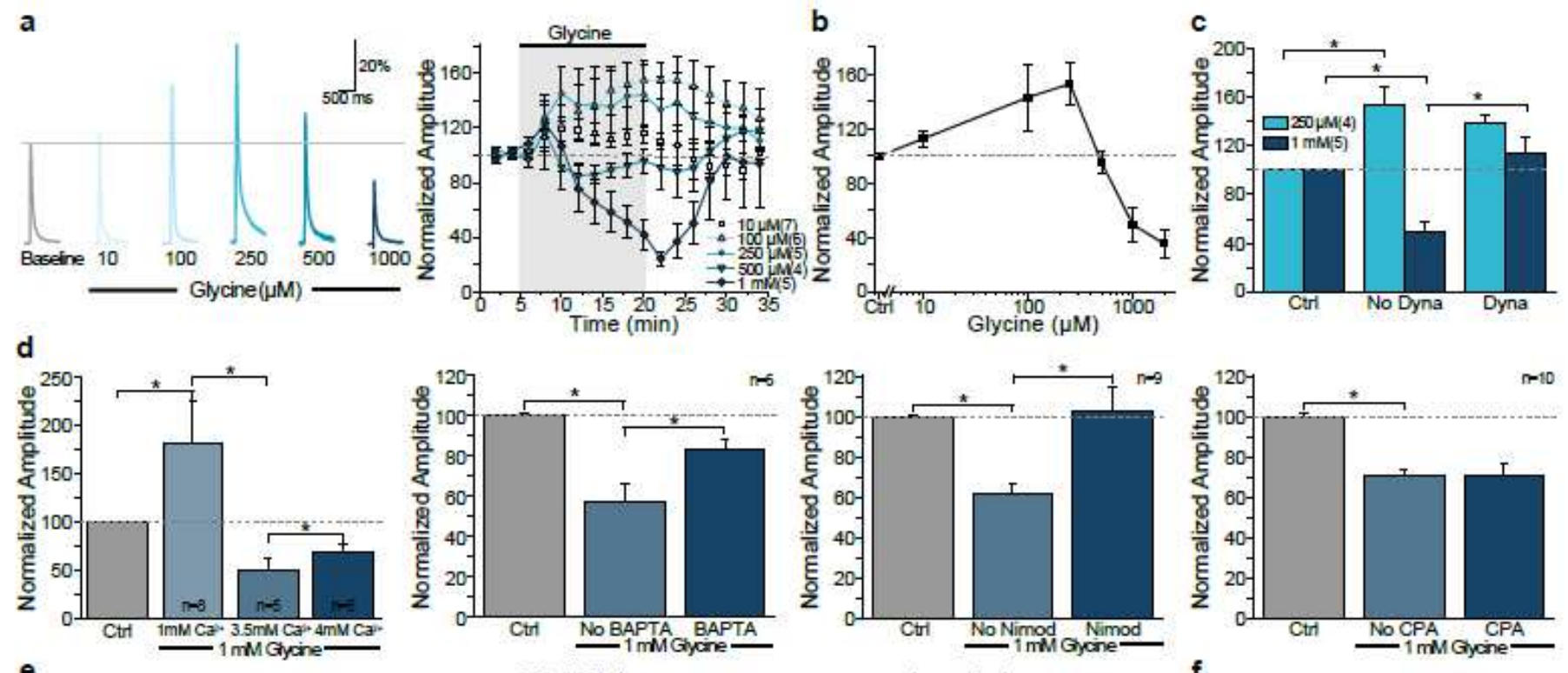

e
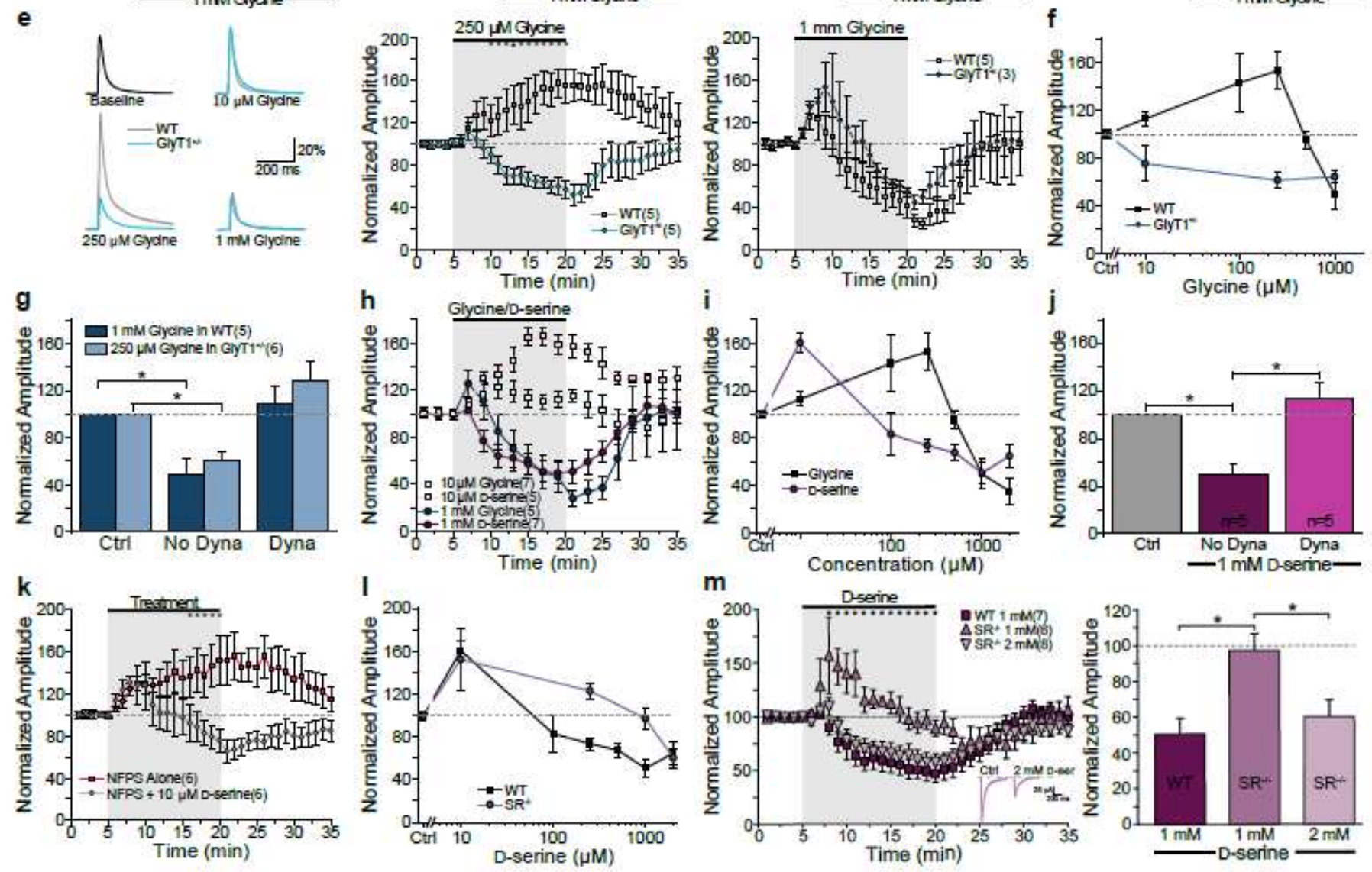

\section{Figure 1}

Increasing exogenous glycine and D-serine concentrations results in NMDAR internalization, which is dependent on $\mathrm{Ca} 2+$ influx. a) Normalized raw traces showing the effect of increasing concentrations of exogenous glycine on Schaffer Collateral NMDAR-EPSCs and mean time-course data showing the effect 
of a 15mins application of various glycine concentrations. b) A dose-response curve of glycine and NMDAR EPSC amplitudes. c) The effect of $250 \mu \mathrm{M}$ and $1 \mathrm{mM}$ glycine on NMDAR-EPSC amplitudes in the presence of dynasore. d) The role of various concentrations of extracellular $\mathrm{Ca} 2+$ on NMDAR EPSC amplitudes in the presence of $1 \mathrm{mM}$ glycine, as well as changing intracellular Ca2+ levels using BAPTA, nimodipine, or CPA. e) The effect of various concentrations of glycine on NMDAR-EPSC amplitudes in GlyT1+/- mice, compared to WT. f) The dose-response curve of the effects of glycine on NMDAR amplitudes in GlyT1+/- mice. g) The effect of $250 \mu \mathrm{M}$ glycine on NMDAR-EPSC amplitudes in GlyT1+/mice in the presence of dynasore. $h)$ The effect of low $(10 \mu \mathrm{M})$ and high $(1 \mathrm{mM})$ concentrations of glycine or D-serine concentrations on NMDAR-EPSC amplitudes. i) The dose-response curve of NMDAR-EPSC amplitudes to $D$-serine compared to glycine. j) The effect of $1 \mathrm{mM} \mathrm{D-serine} \mathrm{in} \mathrm{the} \mathrm{presence} \mathrm{of} \mathrm{dynasore.} k$ ) The effect of $10 \mu \mathrm{M} D$-serine while elevating endogenous glycine levels with NFPS. I) A dose-response curve showing the effect of exogenous D-serine levels on SR-/-mice. $m$ ) The effect of a higher dose of Dserine ( $1 \mathrm{mM}$ vs. $2 \mathrm{mM}$ ) on NMDAR-EPSC amplitudes in SR-/- mice compared to their WT littermates. Data is mean \pm SEM; statistical significance $p<0.05$ * 


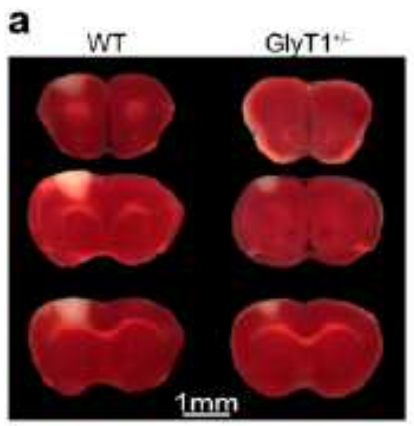

b

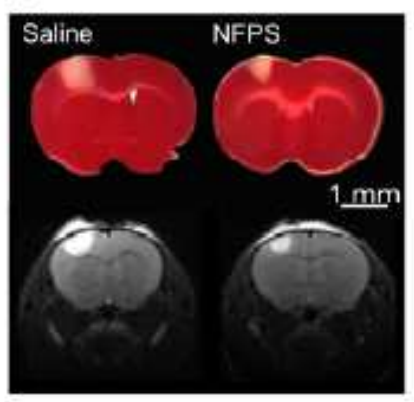

d

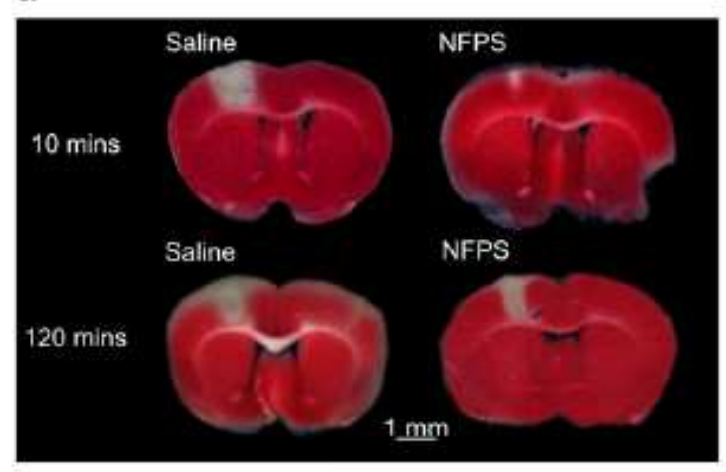

e

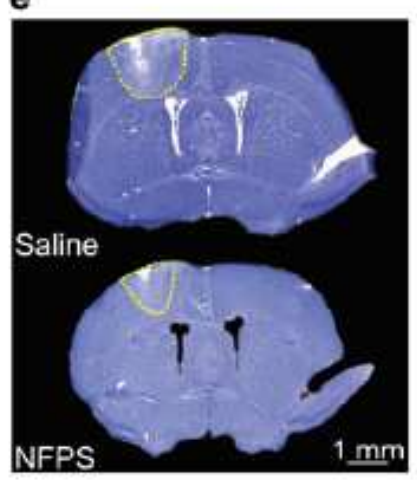

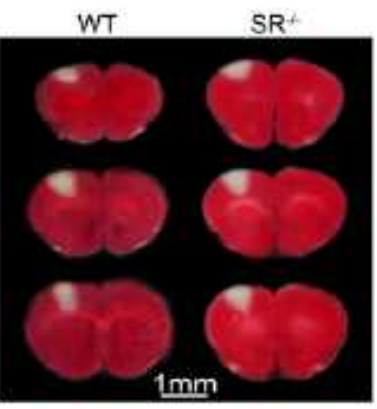
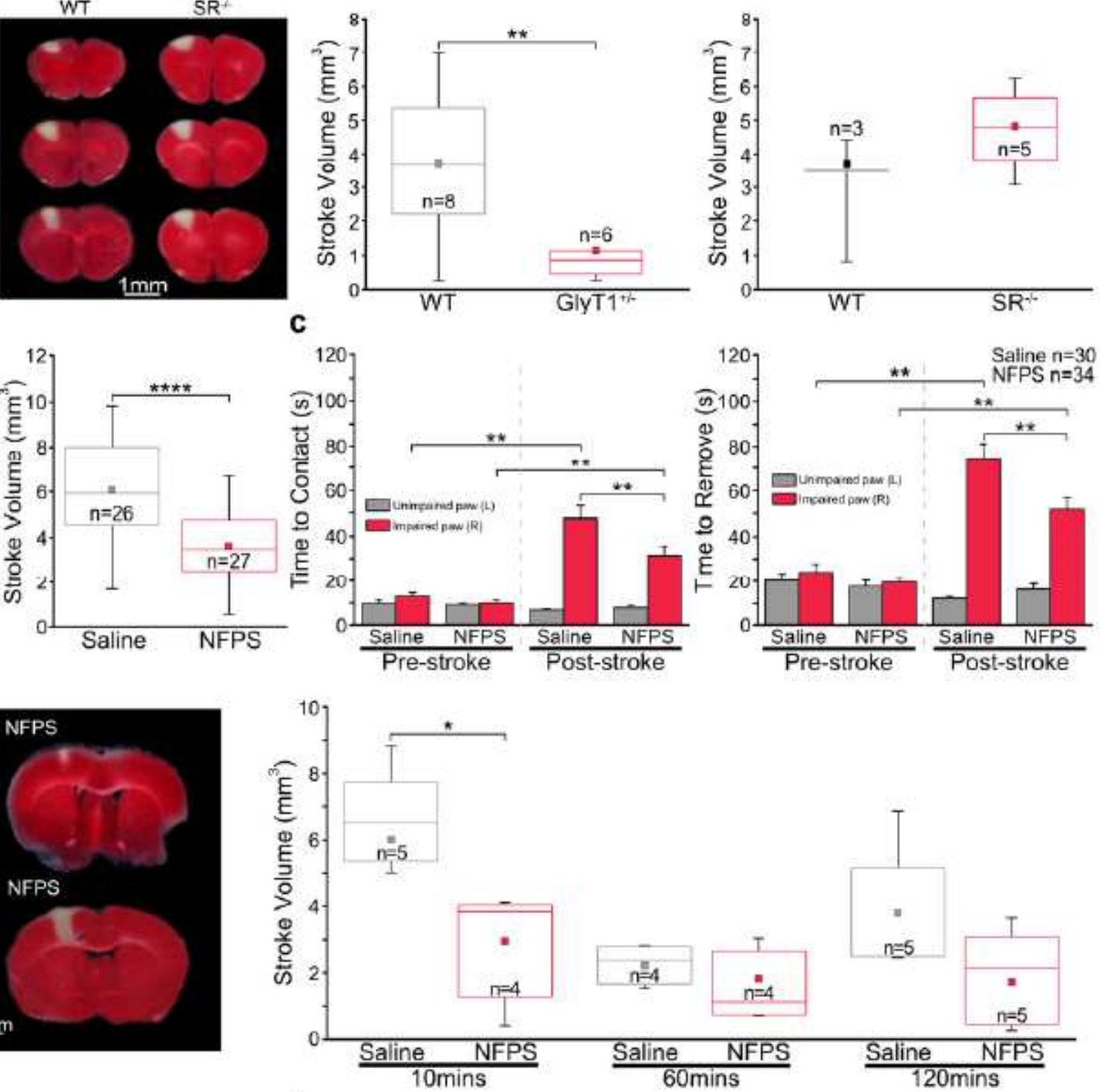

f
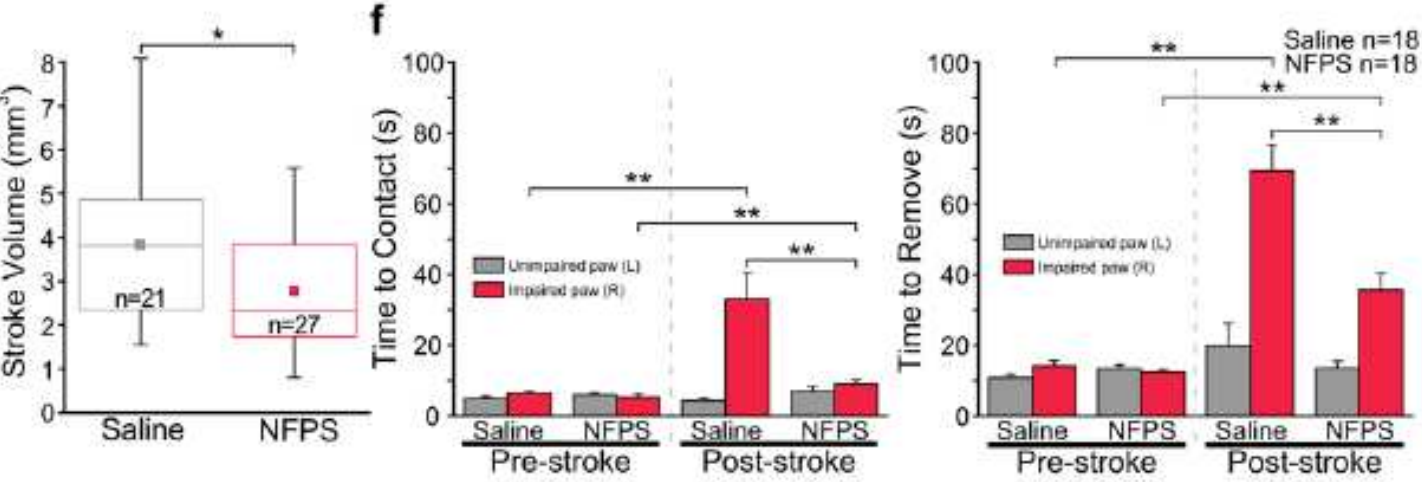

\section{Figure 2}

Elevation of extracellular glycine results in a smaller infarct volume and decreased motor behavioural deficits following photothrombotic and endothelin-1 stroke. a) Representative serial coronal sections of TTC-stained mouse forebrain (slice thickness $500 \mu \mathrm{M}$ ) and their corresponding box and whisker plots showing the infarct volume when assessed $48 \mathrm{hrs}$ after the induction of a unilateral photothrombotic stroke in GlyT1+/- and SR-/- mice relative to WT mice. b) Representative TTC-stained (top) or magnetic 
resonance imaging (bottom) sections showing representative stroke regions observed $48 \mathrm{hrs}$ following the induction of photothrombotic (PT) stroke, and a box and whisker plot showing stroke volume in salinetreated or NFPS-treated mice $24 \mathrm{hrs}$ prior to stroke induction. c) The effect of $24 \mathrm{hrs}$ pre-stroke NFPS administration on post-stroke time to contact and time to remove in the adhesive removal task compared with saline treatment, when evaluated 48hrs following PT stroke. d) Effect of various post-stroke administration time-points of NFPS treatment on stroke volume with their corresponding box and whisker plots following PT stroke. e) Representative cresyl violet sections ( $25 \mu \mathrm{m}$ thick) 48hrs following endothelin-1 (ET-1) stroke obtained from saline-treated and NFPS-treated mice 24hrs prior, in which the extent of the infarct is shown within the yellow border and box and whisker plot depicting infarct volume. f) The effect of $24 \mathrm{hrs}$ pre-stroke NFPS administration on post-stroke time to contact and time to remove in the adhesive removal task compared with saline treatment following ET-1 stroke. Data is mean \pm SEM; statistical significance $p<0.05 *, p<0.01 * *, p<0.001 * \star *$, and $p<0.0001 * \star \star *$. 

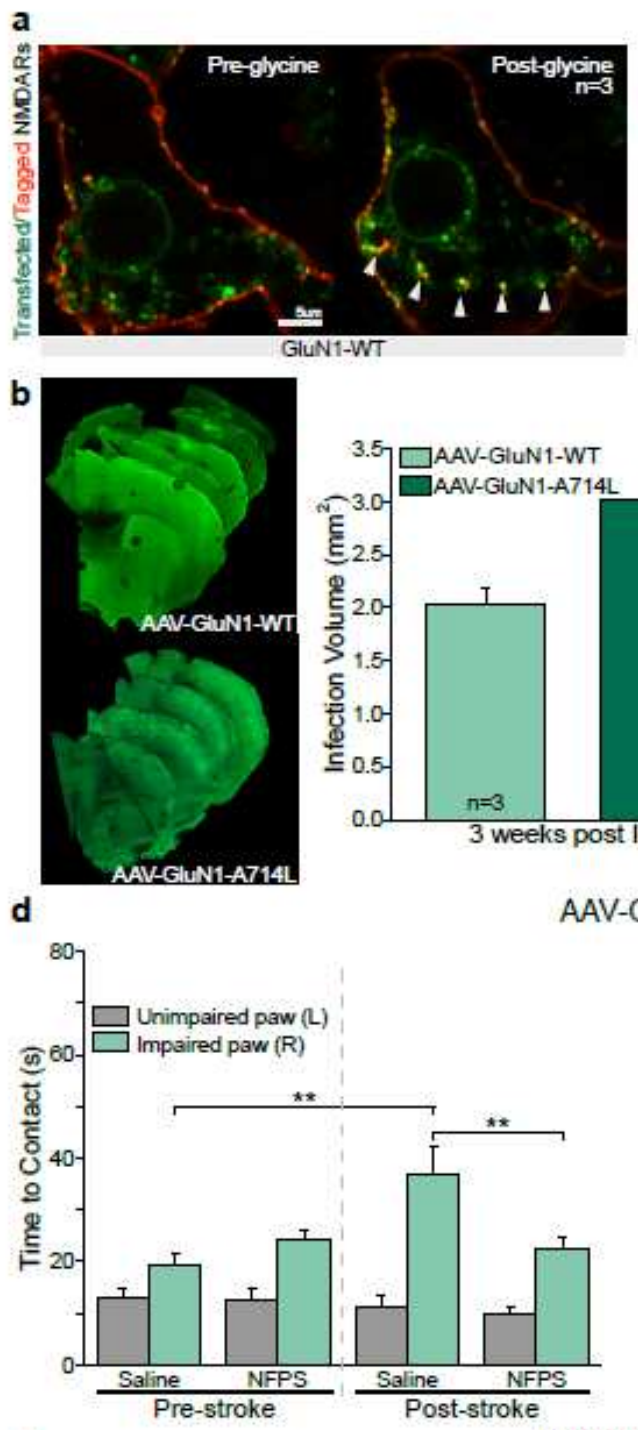

AAV-GluN1-A714L

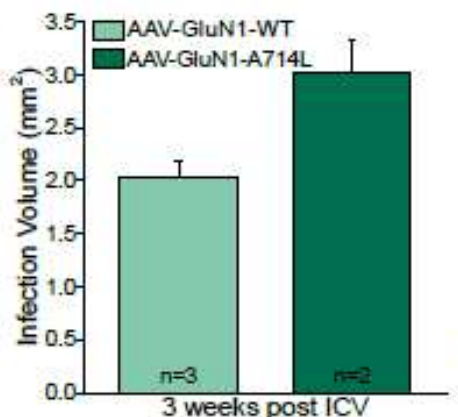

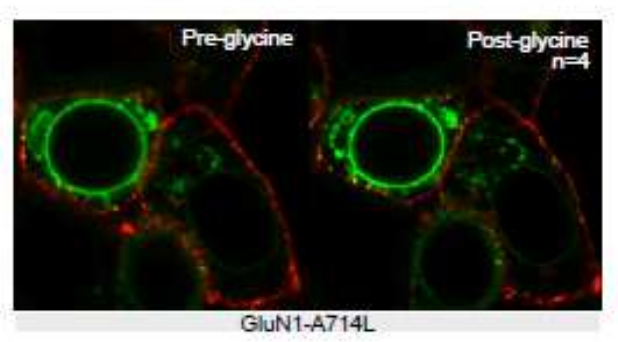

c
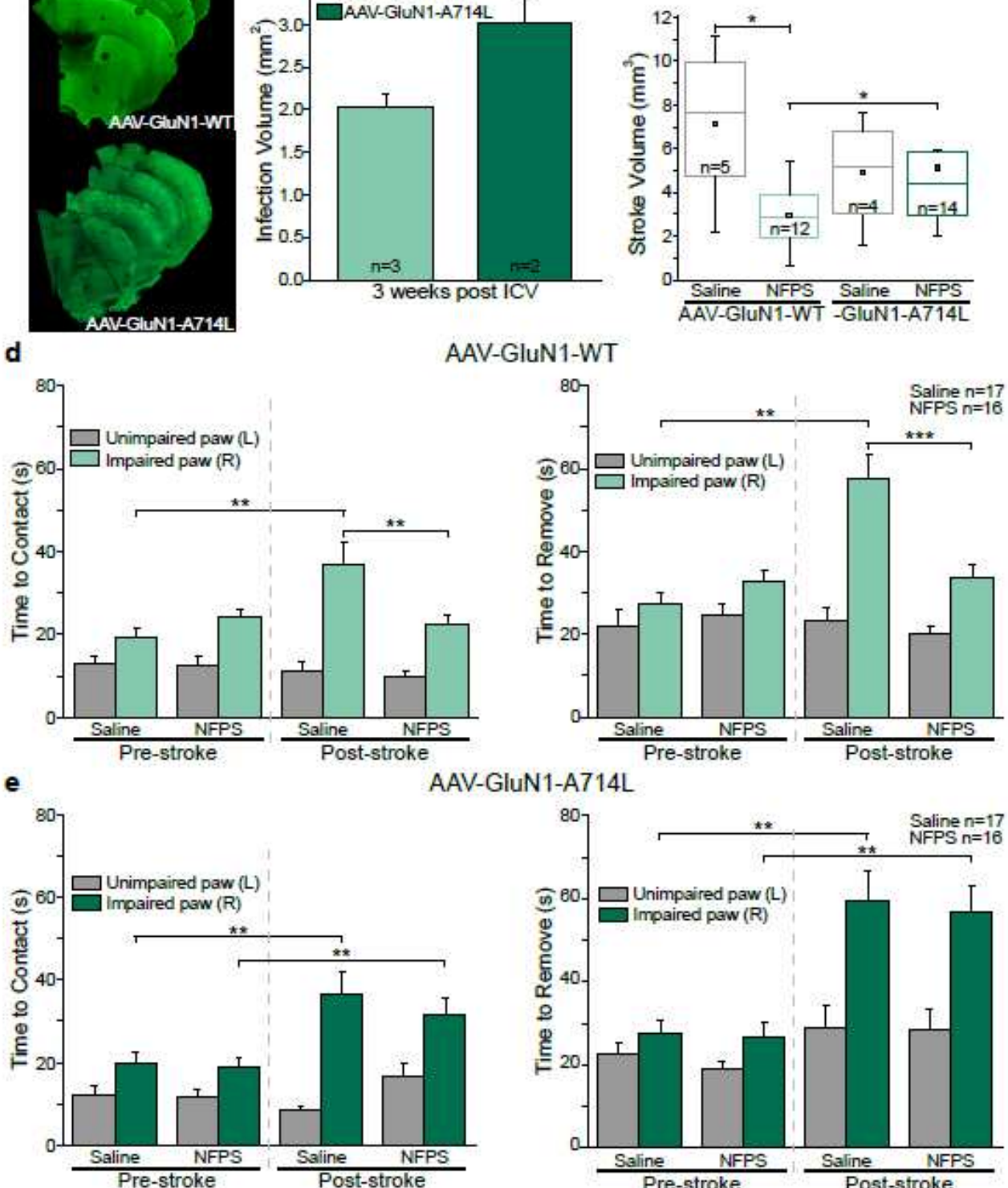

\section{Figure 3}

Infection of the stroke site with the non-internalizing GluN1-A714L mutation abolishes the protective effect of elevating extracellular glycine on stroke volume and during a behavioural task. a) Visual representation of NMDAR internalization in GluN1-WT or GluN1-A714L transfected HEK293 cells following application of $1 \mathrm{mM}$ glycine. Transfected NMDARs are labeled in green, while extracellular NMDARs are additionally labeled with red cell impermeable nanobody staining. b) Representative images 
showing the extent of viral spread in the mouse forebrain following infection between mice infected with AAV-GluN1-WT or AAV-GluN1-A714L. c) Box and whisker plot showing the effect of NFPS administration 24hrs prior to PT stroke induction in mice infected with AAV-GluN1-WT or AAV-GluN1-A714L. d-e) The effect of NFPS on post-stroke time to contact and time to remove in the adhesive removal task compared with saline treatment, in mice infected with AAV-GluN1-WT (d), and in mice infected with AAV-GluN1A714L (e) 48hrs following PT stroke. Data is mean \pm SEM; statistical significance $p<0.05 *, p<0.01$ **, and $p<0.001$ ***.

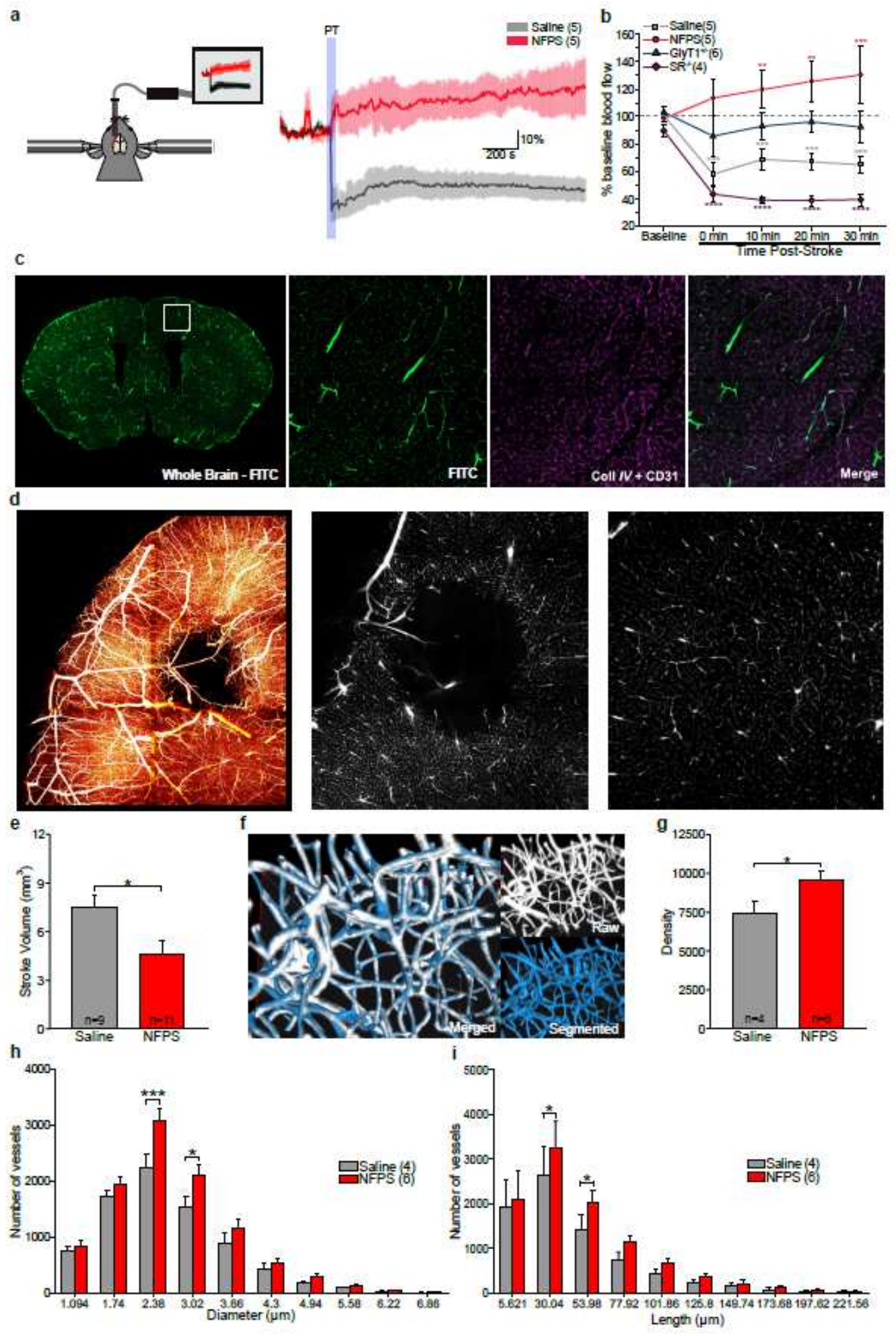




\section{Figure 4}

Laser doppler flowmetry blood flow and automatic segmentation of vascular data with AIVIA 9. a) Diagram depicting laser doppler flowmetry set-up and the measured effect of NFPS on cerebral blood flow following photothrombotic (PT) stroke. b) Time-course of post-stroke cerebral blood flow following PT in mice with varying levels of glycine or D-serine. c) $50 \mu \mathrm{m}$ coronal section of brain perfused with FITCBSA. Magnified images from the sensorimotor cortex demonstrating exact colocalization of FITC-BSA perfusion (green) with CD31 and CollIV vascular immunostaining (purple). d) Colorized max projection of stroked hemisphere, and single section of raw images depicting vasculature at the stroke and below the stroke, acquired with a light sheet microscope 48hrs following PT stroke. e) Stroke volume bar graph in saline- or NFPS-treated mice, calculated by an automated deep learning prediction model. f) Merged image demonstrating exact colocalization of AIVIA's automatic segmentation to raw data. g) Density of vessels in peri infarct region in saline- or NFPS-treated mice. $\mathrm{h}-\mathrm{i}$ ) Number of vessels in the stroke area according to diameter and to length. Data is mean \pm SEM; statistical significance $p<0.05 *, p<0.01 * \star, p$ $<0.001 * \star \star$, and $p<0.0001 * \star \star \star$.

\section{Supplementary Files}

This is a list of supplementary files associated with this preprint. Click to download.

- Supplementarymethods.pdf

- SupplementalVideo1.gif

- SupplementalVideo2.gif

- SupplementalVideo3.mp4

- SupplementalVideo4.mp4 